The Astrophysical JournaL, 547:12-26, 2001 January 20

(C) 2001. The American Astronomical Society. All rights reserved. Printed in U.S.A.

\title{
QUASAR CLUSTERING AND THE LIFETIME OF QUASARS
}

\author{
Paul Martini ${ }^{1}$ and David H. Weinberg \\ Department of Astronomy, 140 West 18th Avenue, Ohio State University, Columbus, OH 43210; martini@astronomy.ohio-state.edu, \\ dhw@astronomy.ohio-state.edu \\ Received 2000 February 21 ; accepted 2000 September 21
}

\begin{abstract}
Although the population of luminous quasars rises and falls over a period of $\sim 10^{9} \mathrm{yr}$, the typical lifetime of individual quasars is uncertain by several orders of magnitude. We show that quasar clustering measurements can substantially narrow the range of possible lifetimes, with the assumption that luminous quasars reside in the most massive host halos. If quasars are long-lived, then they are rare phenomena that are highly biased with respect to the underlying dark matter, while if they are shortlived they reside in more typical halos that are less strongly clustered. For a given quasar lifetime, we calculate the minimum host halo mass by matching the observed space density of quasars, using the Press-Schechter approximation. We use the results of Mo \& White to calculate the clustering of these halos, and hence of the quasars they contain, as a function of quasar lifetime. A lifetime of $t_{Q}=4 \times 10^{7}$ yr, the $e$-folding timescale of an Eddington luminosity black hole with accretion efficiency $\epsilon=0.1$, corresponds to a quasar correlation length of $r_{0} \approx 10 h^{-1} \mathrm{Mpc}$ in low-density cosmological models at $z=2-3$; this value is consistent with current clustering measurements, but these have large uncertainties. High-precision clustering measurements from the Two-Degree Field (2dF) and Sloan quasar surveys will test our key assumption of a tight correlation between quasar luminosity and host halo mass, and if this assumption holds, then they should determine $t_{Q}$ to a factor of 3 or better. An accurate determination of the quasar lifetime will show whether supermassive black holes acquire most of their mass during highluminosity accretion, and it will show whether the black holes in the nuclei of typical nearby galaxies were once the central engines of high-luminosity quasars.
\end{abstract}

Subject headings: dark matter - large-scale structure of universe - quasars: general

\section{INTRODUCTION}

Mounting evidence for the existence of supermassive black holes in the centers of nearby galaxies (recently reviewed by, e.g., Richstone et al. 1998) supports the longstanding hypothesis that quasars are powered by black hole accretion (e.g., Salpeter 1964; Zeldovich \& Novikov 1964; Lynden-Bell 1969). However, one of the most basic properties of quasars, the typical quasar lifetime, $t_{Q}$, remains uncertain by orders of magnitude. The physics of gravitational accretion and radiation pressure provides one natural timescale, the $e$-folding time $t_{e}=M_{\mathrm{BH}} / \dot{M}=4$ $\times 10^{8} \mathrm{\epsilon l} \mathrm{yr}$ of a black hole accreting mass with a radiative efficiency $\epsilon=L / \dot{M} c^{2}$ and shining at a fraction $l=L / L_{\mathrm{E}}$ of its Eddington luminosity (Salpeter 1964). However, while $\epsilon \sim 0.1$ and $l \sim 1$ are plausible values for a quasar, it is possible that black holes accrete much of their mass while radiating at much lower efficiency, or at a small fraction of $L_{\mathrm{E}}$. The task of determining $t_{Q}$ must therefore be approached empirically.

The observed evolution of the quasar luminosity function imposes a strong upper limit on $t_{Q}$ of about $10^{9} \mathrm{yr}$, since the whole quasar population rises and falls over roughly this interval (see, e.g., Osmer 1998). The lifetime of individual quasars could be much shorter than the lifetime of the quasar population, however, and lower limits of $t_{Q} \sim 10^{5} \mathrm{yr}$ rest on indirect arguments, such as the requirement that quasars maintain their ionizing luminosity long enough to explain the proximity effect in the Ly $\alpha$ forest (e.g., Bajtlik, Duncan, \& Ostriker 1988; Bechtold 1994). A typical lifetime

\footnotetext{
${ }^{1}$ Current address: Carnegie Observatories, 813 Santa Barbara Street, Pasadena, CA 91101; martini@ociw.edu.
}

of $t_{Q} \sim 10^{9}$ yr would imply that quasars are rare phenomena, arising in at most a small fraction of high-redshift galaxies. Conversely, a lifetime as low as $t_{Q} \sim 10^{5}$ yr would imply that quasars are quite common, suggesting that a large fraction of present-day galaxies went through a brief quasar phase in their youth.

The comoving space density $\Phi(z)$ of active quasars at redshift $z$ is proportional to $t_{Q} n_{H}(z)$, where $n_{H}$ is the comoving space density of quasar hosts. "Demographic" studies of the local black hole population (e.g., Magorrian et al. 1998; Salucci et al. 1999; van der Marel 1999) have opened up one route to determining the typical quasar lifetime: counting the present-day descendants of the quasar central engines in order to estimate $n_{H}(z)$ and thus constrain $t_{Q}$ by matching $\Phi(z)$. Roughly speaking, the ubiquity of black holes in nearby galaxies suggests that quasars are common and that $t_{Q}$ is likely in the range of $10^{6}-10^{7}$ (e.g., Richstone et al. 1998; Haehnelt, Natarajan, \& Rees 1998; Salucci et al. 1999). However, as Richstone et al. (1998) emphasize, the lifetime estimated in this way depends crucially on the way one links the mass of a present-day black hole to the luminosity of a high-redshift quasar, which in turn depends on assumptions about the growth of black hole masses since the quasar epoch via mergers or lowefficiency accretion.

In this paper we propose an alternative route to the quasar lifetime, using measurements of high-redshift quasar clustering. The underlying idea goes back to the work of Kaiser (1984) and Bardeen et al. (1986): in models of structure formation based on gravitational instability of Gaussian primordial fluctuations, the rare, massive objects are highly biased tracers of the underlying mass distribution, while more common objects are less strongly biased. There- 
fore, a longer quasar lifetime $\left(t_{Q}\right)$ should imply a more clustered quasar population, provided that luminous quasars reside in massive hosts. The specific calculations that we present in this paper use the Press \& Schechter (1974; hereafter PS) approximation for the mass function of dark matter halos and the Mo \& White (1996, hereafter MW) and Jing (1998) approximations for the bias of these halos as a function of mass. The path from clustering to quasar lifetime has its own uncertainties; in particular, our predictions for quasar clustering rely on the assumption that the luminosity of a quasar during its active phase is a monotonically increasing function of the mass of its host dark matter halo. However, the assumptions in the clustering approach are at least very different from those in the black hole mass function approach, and they can be tested empirically by detailed studies of quasar clustering as a function of luminosity and redshift.

Our theoretical model of quasar clustering follows a general trend in which the study of quasar activity is embedded in the broader context of galaxy formation and gravitational growth of structure (e.g., Efstathiou \& Rees 1988; Turner 1991; Haehnelt \& Rees 1993; Katz et al. 1994; Haehnelt et al. 1998; Haiman \& Loeb 1998; Monaco, Salucci, \& Danese 2000; Kauffmann \& Haehnelt 2000). This paper also continues a theme that is prominent in recent work on the clustering of Lyman-break galaxies, namely, that the clustering of high-redshift objects is a good tool for understanding the physics of their formation and evolution (e.g., Adelberger et al. 1998; Katz, Hernquist, \& Weinberg 1999; Kolatt et al. 1999; Mo, Mao, \& White 1999). Our model of the quasar population is idealized, but by focusing on a simple calculation with clearly defined predictions, we hope to highlight the link between quasar lifetime and clustering strength. After presenting the theoretical results, we draw some inferences from existing estimates of the quasar correlation length. However, our study is motivated mainly by an anticipation of vastly improved measurements of quasar clustering from the Two-Degree Field (2dF) and Sloan quasar surveys (see, e.g., Boyle et al. 1999; Fan et al. 1999; York et al. 2000). These measurements can test various hypotheses about the origin of quasar activity, including our primary assumption of a monotonic relation between quasar luminosity and host halo mass. If this assumption proves valid, then the first major physical result to emerge from the $2 \mathrm{dF}$ and Sloan measurements of high-redshift quasar clustering will be a new determination of the typical quasar lifetime.

\section{METHOD}

\subsection{Overview}

We adopt a simple model of the high-redshift quasar population that is, doubtless, idealized, but which should be reasonably accurate for our purpose of computing clustering strength as a function of quasar lifetime. We assume that all quasars reside in dark matter halos and that a given halo hosts at most one active quasar at a time. The first assumption is highly probable, since a dark matter collapse is necessary to seed the growth of a black hole, and the second should be a fair approximation at high redshift, where the masses of large halos are comparable to the masses of individual galaxy halos today.

Our strongest and most important assumption is that the luminosity of a quasar during its active phase is monotoni- cally related to the mass of its host dark matter halo, and that all sufficiently massive halos host an active quasar at some point. More precisely, we assume that an absolutemagnitude-limited sample of quasars at redshift $z$ samples the most massive halos present at that redshift, and that the probability that a halo above the minimum host mass $M_{\text {min }}$ harbors an active quasar at any given time is $t_{Q} / t_{H}$, where $t_{Q}$ is the average quasar lifetime and $t_{H}$ is the halo lifetime. We can therefore compute the value of $M_{\min }$ for a quasar population with comoving space density $\Phi(z)$ from the condition

$$
\Phi(z)=\int_{M_{\min }}^{\infty} d M n(M) \frac{t_{Q}}{t_{H}} .
$$

We compute $n(M)$ using the PS approximation, and we compute the bias of halos with $M>M_{\min }$ using the M-W approximation.

A connection between quasar luminosity and host halo mass is plausible on theoretical grounds: the cores of massive halos collapse early, giving black holes time to grow, and these halos provide larger gas supplies for fueling activity. It is also plausible on empirical grounds: local black hole masses are correlated with the host spheroid luminosity (Magorrian et al. 1998; van der Marel 1999; Salucci et al. 1999), which in turn is correlated with stellar velocity dispersion (Faber \& Jackson 1976). A precisely monotonic relation is certainly an idealization, and we explore the effects of relaxing this assumption in $\S$ 4.1. The assumption of an approximately monotonic relation can be tested empirically by searching for the predicted relation between clustering strength and luminosity, as we discuss in $\S 4.3$.

The ubiquity of black holes in luminous local spheroids supports our assumption that all sufficiently massive halos go through a quasar phase. However, once the quasar space density declines at $z<2$, the occurrence of quasar activity must be determined by fueling rather than by the mere existence of a massive black hole, so it is not plausible that all large halos host a low-redshift quasar. We therefore apply our model only to the high-redshift quasar population, at $z \geq 2$.

We implicitly assume that a quasar turns on at a random point in the life of its host halo. In this sense, our model differs subtly from that of Haehnelt et al. (1998), who assume that a quasar turns on when the halo is formed, but this difference is unlikely to have a significant effect on the predicted clustering. Haehnelt et al. (1998) pointed out that a longer quasar lifetime would correspond to stronger quasar clustering because of the association with rarer peaks of the mass distribution, but they did not calculate this relation in detail.

Because the quasar lifetime enters our calculation only through the probability $t_{Q} / t_{H}$ that a halo hosts an active quasar at a given time, it makes no difference whether the quasar shines continuously or turns on and off repeatedly with a short duty cycle (as argued recently by Ciotti \& Ostriker 1999). For our purposes, $t_{Q}$ is the total time that the quasar shines at close to its peak luminosity. We also assume that quasars radiate isotropically, with a beaming factor $f_{B}=1$, but because a smaller beaming factor simply changes the conversion between observed surface density and intrinsic comoving space density, all our results can be scaled to smaller average beaming factors by replacing $t_{Q}$ with $f_{B} t_{Q}$. 


\subsection{Notation}

All our calculations assume Gaussian primordial fluctuations. We denote by $P(k)$ the power spectrum of these fluctuations as extrapolated to the present day $(z=0)$ by linear theory. The rms fluctuation of the linear density field on mass scale $M$ is

$$
\sigma(M)=\left[\frac{1}{2 \pi^{2}} \int_{0}^{\infty} d k k^{2} P(k) \tilde{W}^{2}(k r)\right]^{1 / 2},
$$

where

$$
\tilde{W}(k r)=\frac{3(k r \sin k r-\cos k r)}{(k r)^{3}}, \quad r=\left(\frac{3 M}{4 \pi \rho_{0}}\right)^{1 / 3},
$$

is the Fourier transform of a spherical top-hat containing average mass $M$. The mean density of the universe at $z=0$ is $\rho_{0}=2.78 \times 10^{11} \Omega_{M} h^{2} M_{\odot} \mathrm{Mpc}^{-3}$, with $h \equiv H_{0} /(100$ $\left.\mathrm{km} \mathrm{s}^{-1} \mathrm{Mpc}^{-1}\right)$. The rms fluctuation can be considered as a function of either the mass scale $M$ or the equivalent radius $r$. We define the normalization of the power spectrum by the value of $\sigma_{8} \equiv \sigma\left(r=8 h^{-1} \mathrm{Mpc}\right)$. is

The rms fluctuation of the linear density field at redshift $z$

$$
\sigma(M, z)=\sigma(M) D(z),
$$

where $D(z)$ is the linear growth factor $D(z)$, defined such that $D(z=0)=1$. The general expression for the growth factor in terms of the scaled expansion factor $y=(1+z)^{-1}$ is

$$
\delta(y)=\frac{5}{2} \Omega_{M} \frac{1}{y} \frac{d y}{d \tau} \int_{0}^{y}\left(\frac{d y^{\prime}}{d \tau}\right)^{-3} d y^{\prime},
$$

where $D(y)=\delta(y)$ for $\Omega_{M}=1, D(y)=\delta(y) / \delta(1)$ for $\Omega_{M}<1$, and the dimensionless time variable is $\tau=H_{0} t$ (Heath 1977; Carroll, Press, \& Turner 1992). If the dominant energy components are pressureless matter and a cosmological constant with $\Omega_{\Lambda}=\Lambda / 3 H_{0}^{2}$, then the Friedmann equation implies

$$
\left(\frac{d y}{d \tau}\right)^{2}=1+\Omega_{M}\left(y^{-1}-1\right)+\Omega_{\Lambda}\left(y^{2}-1\right) .
$$

For an $\Omega_{M}=1, \Omega_{\Lambda}=0$ universe, $D(z)=(1+z)^{-1}$. Peebles (1980, eq. [11.16]) gives an exact analytic expression for $D(z)$ for the case $\Omega_{M}<1, \Omega_{\Lambda}=0$, and Carroll et al. (1992, eq. [29]) give an accurate analytic approximation for $\Omega_{\Lambda} \neq$ 0 . In our notation, $\sigma(M)$ without any explicit $z$ always refers to the rms linear mass fluctuation on scale $M$ at $z=0$.

At any redshift, we can define a characteristic mass $M_{*}(z)$ by the condition

$$
\sigma\left[M_{*}(z)\right]=\delta_{c}(z)=\frac{\delta_{c, 0}}{D(z)},
$$

where $\delta_{c}(z)$ is the threshold density for collapse of a homogeneous spherical perturbation at redshift $z$. Because we implicitly define the density field as "existing" at $z=0$, the collapse threshold $\delta_{c}(z)$ increases with increasing redshift. For an $\Omega_{M}=1, \Omega_{\Lambda}=0$ universe, $\delta_{c, 0}=0.15(12 \pi)^{2 / 3} \approx 1.69$ (see, e.g., Peebles 1980, $\S 19$ ). For other models, we incorporate the dependence of $\delta_{c, 0}$ on $\Omega_{M}$ in Appendix A of Navarro, Frenk, \& White (1997), but because $\Omega_{M}$ approaches one at high redshift in all models, this correction to $\delta_{c}$ is less than $2 \%$ in all the cases that we consider.

\subsection{From the Quasar Lifetime to the Minimum Halo Mass}

For a specified quasar lifetime, we compute the minimum halo mass by matching the comoving number density, $\Phi(z)$, of observed quasars, accounting for the fact that only a fraction $t_{Q} / t_{H}$ of host halos will have an active quasar at the time of observation. The matching condition is equation (1), or, putting in explicit mass and redshift dependences,

$$
\Phi(z)=\int_{M_{\min }}^{\infty} d M \frac{t_{Q}}{t_{H}(M, z)} n(M, z) .
$$

If $t_{Q}>t_{H}(M, z)$, we set the factor $t_{Q} / t_{H}$ to unity. For the halo number density, we use the P-S approximation,

$$
\begin{aligned}
n(M, z) d M= & -\sqrt{\frac{2}{\pi}} \frac{\rho_{0}}{M} \frac{\delta_{c}(z)}{\sigma^{2}(M)} \frac{d \sigma(M)}{d M} \\
& \times \exp \left[-\frac{\delta_{c}^{2}(z)}{2 \sigma^{2}(M)}\right] d M,
\end{aligned}
$$

where $\rho_{0}$ is the mean density of the universe at $z=0, \sigma(M)$ is the rms fluctuation given by equation (2), and $\delta_{c}(z)$ is the critical density for collapse by redshift $z$.

In a gravitational clustering model of structure formation, halos are constantly growing by accretion and mergers, so the definition of a "halo lifetime" is somewhat ambiguous. For $\Omega_{M}(z) \approx 1$, a typical halo survives for roughly a Hubble time before being incorporated into a substantially larger halo, since the age of the universe at redshift $z$ is also the characteristic dynamical time of objects forming at that redshift. Thus, to a first approximation, one could simply substitute $t_{H}(M, z)=t_{U}(z)$ in equation (9). We can do somewhat better by using the extended PressSchechter formalism (e.g., Bond et al. 1991; Lacey \& Cole 1993) to calculate the average halo lifetime, thereby accounting for the dependence of $t_{H}$ on the power spectrum shape and the halo mass. Structure grows more rapidly in a cosmology with a redder power spectrum, and more massive halos accrete mass more rapidly.

Equation (2.22) of Lacey \& Cole (1993) gives the probability that a halo of mass $M_{1}$ existing at time $t_{1}$ will have been incorporated into a new halo of mass greater than $M_{2}$ by time $t_{2}$ :

$$
\begin{aligned}
P\left(S<S_{2}, \omega_{2} \mid S_{1}, \omega_{1}\right)= & \frac{1}{2} \frac{\left(\omega_{1}-2 \omega_{2}\right)}{\omega_{1}} \exp \left[\frac{2 \omega_{2}\left(\omega_{1}-\omega_{2}\right)}{S_{1}}\right] \\
& \times\left\{1-\operatorname{erf}\left[\frac{S_{2}\left(\omega_{1}-2 \omega_{2}\right)+S_{1} \omega_{2}}{\sqrt{2 S_{1} S_{2}\left(S_{1}-S_{2}\right)}}\right]\right\} \\
+ & \frac{1}{2}\left\{1-\operatorname{erf}\left[\frac{S_{1} \omega_{2}-S_{2} \omega_{1}}{\sqrt{2 S_{1} S_{2}\left(S_{1}-S_{2}\right)}}\right]\right\}, \quad(10)
\end{aligned}
$$

where $S_{1}=\sigma^{2}\left(M_{1}\right), S_{2}=\sigma^{2}\left(M_{2}\right), \omega_{1}=\delta_{c}\left(t_{1}\right)$, and $\omega_{2}=$ $\delta_{c}\left(t_{2}\right)$. In this equation, $\omega$ plays the role of the "time" variable, with $\omega_{2}<\omega_{1}$ corresponding to $t_{2}>t_{1}$, and $S$ plays the role of the "mass" variable, with $S_{2}<S_{1}$ corresponding to $M_{2}>M_{1}$. For a halo of mass $M$ existing at time $t_{U}(z)$, we define the halo lifetime to be the median interval before such a halo is incorporated into a halo of mass $2 M$. Thus, $t_{H}(M, z)=\hat{t}_{S}-t_{U}(z)$, where $\hat{t}_{S}$ is the time at which the probability in equation (10) equals 0.5 , for $S_{1}=\sigma^{2}(M)$ and $S_{2}=$ $\sigma^{2}(2 M)$. Clearly, other plausible definitions of $t_{H}(M, z)$ are possible, and they would give answers different by factors of order unity. With our definition, a black hole that lights up 
repeatedly is considered the "same" quasar as long as the mass of its host halo remains the same within a factor of 2 . If the host merges into a much larger halo and the black hole lights up again, it is considered a "new" quasar. We show the halo lifetimes for different masses and power spectra when we discuss specific models below.

For comoving space densities $\Phi(z)$, we adopt values based on the work of Boyle et al. (1990), Hewett, Foltz, \& Chaffee (1993), and Warren, Hewett, \& Osmer (1994). Since observations constrain the number of objects per unit redshift per unit solid angle, the conversion to comoving space density depends on the values of the cosmological parameters. We provide the formulae for these conversions in the Appendix, and in Table A1 we list our adopted values of $\Phi(z)$ and the surface densities of objects to which these space densities correspond. In general, $\Phi(z)$ represents the space density of quasars above some absolute magnitude, corresponding to a surface density above some apparent magnitude. In $\S 4.3$ we discuss how to scale our results to predict the clustering of samples with different measured surface densities.

\subsection{From Minimum Halo Mass to Clustering Length}

Halos with $M>M_{*}$ are clustered more strongly than the underlying distribution of mass. MW give an approximate formula,

$$
b(M, z)=1+\frac{1}{\delta_{c, 0}}\left[\frac{\delta_{c}^{2}(z)}{\sigma^{2}(M)}-1\right],
$$

for the bias factor of halos of mass $M$ at redshift $z$. On large scales, the ratio of rms fluctuations in halo number density to rms fluctuations in mass should be $b(M, z)$. This formula is derived from an extended Press-Schechter analysis, and it agrees fairly well with the results of $N$-body simulations on scales where the rms mass fluctuations are less than unity. The MW formula becomes less accurate for halos with $M<M_{*}$, i.e., $\sigma(M)<\delta_{c}(z)$, and Jing (1998) provides an empirical correction that fits the $N$-body results,

$$
\begin{aligned}
b(M, z)= & \left\{1+\frac{1}{\delta_{c, 0}}\left[\frac{\delta_{c}^{2}(z)}{\sigma^{2}(M)}-1\right]\right\} \\
& \times\left[\frac{\sigma^{4}(M)}{2 \delta_{c}^{4}(z)}+1\right]^{\left(0.06-0.02 n_{\text {eff }}\right)},
\end{aligned}
$$

where $n_{\text {eff }}=3-6(d \ln \sigma / d \ln M)$ is the effective index of the power spectrum on a mass scale $M$.

According to our model, the quasars at redshift $z$ only reside in halos of mass $M>M_{\text {min }}$. The effective bias of these host halos is the bias factor (eq. [12]) weighted by the number density and lifetime of the corresponding halos:

$$
\begin{aligned}
b_{\text {eff }}\left(M_{\min }, z\right)= & \int_{M_{\min }}^{\infty} d M \frac{b(M, z) n(M, z)}{t_{H}(M, z)} \\
& \times\left[\int_{M_{\min }}^{\infty} d M \frac{n(M, z)}{t_{H}(M, z)}\right]^{-1} .
\end{aligned}
$$

Because the halo number density drops steeply with increasing mass, the effective bias is usually only slightly larger than the bias factor at the minimum halo mass, $b\left(M_{\min }, z\right)$.

As our measure of clustering amplitude, we use the radius $r_{1}$ of a top-hat sphere in which the rms fluctuation $\sigma_{Q}$ of quasar number counts (in excess of Poisson fluctuations) is unity. This quantity is similar to the correlation length $r_{0}$ at which the quasar correlation function $\xi(r)$ is unity, but it can be more robustly constrained observationally because it does not require fitting the scale-dependence of $\xi(r)$. For a power-law correlation function $\xi(r)=\left(r / r_{0}\right)^{-1.8}, r_{1} \approx 1.4 r_{0}$. With our adopted approximation for the bias, $r_{1}$ is determined implicitly by the condition

$$
\sigma_{Q}\left(r_{1}, z\right)=b_{\text {eff }}\left(M_{\min }, z\right) \sigma\left(r_{1}\right) D(z)=1,
$$

where $\sigma\left(r_{1}\right)$ is the rms linear mass fluctuation at $z=0$ in spheres of radius $r_{1}$. For a specified cosmology, mass power spectrum $P(k)$, quasar lifetime $t_{Q}$, and comoving space density $\Phi(z)$, we determine $r_{1}$ from equation (14), computing $\sigma(r)$ from equation (2), $D(z)$ from equation (5), $M_{\min }$ from equation (8), and $b_{\text {eff }}\left(M_{\min }\right)$ from equations (12) and (13).

\subsection{Results for Power-Law Power Spectra}

Models with a power-law power spectrum, $P(k) \propto k^{n}$, provide a useful illustration of our methods, since many steps of the calculation can be done analytically. For such models, the dependence of rms fluctuation on mass is also a power law,

$$
\begin{aligned}
\sigma(M)=\frac{\sigma(M, z)}{D(z)} & =\frac{\delta_{c, 0}}{D(z)}\left[\frac{M}{M_{*(z)}}\right]^{-(3+n) / 6} \\
& =\delta_{c}(z)\left[\frac{M}{M_{*(z)}}\right]^{-(3+n) / 6},
\end{aligned}
$$

where $M_{*}(z)$ is the characteristic nonlinear mass defined by equation (7). With this substitution, the PS mass function can be expressed as a function of $M_{*}(z)$ and the dimensionless mass variable $x=M / M_{*}(z)$. Integrating to obtain the comoving number density of objects with mass $M>M_{\text {min }}$ yields

$$
\begin{aligned}
N(M> & \left.M_{\min }\right)=\sqrt{\frac{2}{\pi}}\left(\frac{n+3}{6}\right)\left(\frac{\rho_{0}}{M_{*}}\right) \\
& \times \int_{x_{\min }}^{\infty} d x x^{(n-9) / 6} \exp \left[-\frac{1}{2} x^{(n+3) / 3}\right] .
\end{aligned}
$$

For power-law models with $\Omega_{M}=1$, the ratio of the halo lifetime $t_{H}(M, z)$ to the age of the universe at redshift $z$ depends only on $M / M_{*}(z)$ and has no separate dependence on redshift. Figure 1 shows $t_{H}$ and the ratio $t_{H} / t_{U}$ as a function of $M / M_{*}$ for power-law models with $n=0,-1$, and -2 . More massive halos tend to accrete mass more quickly and therefore have shorter median lifetimes. At a given value of $M / M_{*}$, the halo lifetime is shorter for lower $n$ because a greater amount of large-scale power causes the typical mass scale of nonlinear structure to grow more rapidly. Although the calculation of the median halo lifetime via equation (10) is moderately complicated, the median lifetime for large masses asymptotically approaches a constant value

$$
t_{H}(M, z)=\left[2^{(3+n) / 2}-1\right] t_{U}(z), \quad M \gg M_{*}(z), \Omega_{M}=1,
$$

(Lacey \& Cole 1993). We find below that the predicted masses of quasar host halos are indeed in this asymptotic regime for the most plausible parameter choices. The halo lifetime is longer for $\Omega_{M}<1$ than for $\Omega_{M}=1$ because fluctuations grow more slowly in a low-density universe, but $t_{H}$ still asymptotically approaches a constant value. The dotted curves in Figure 1 illustrate the case of $n=-1$, 


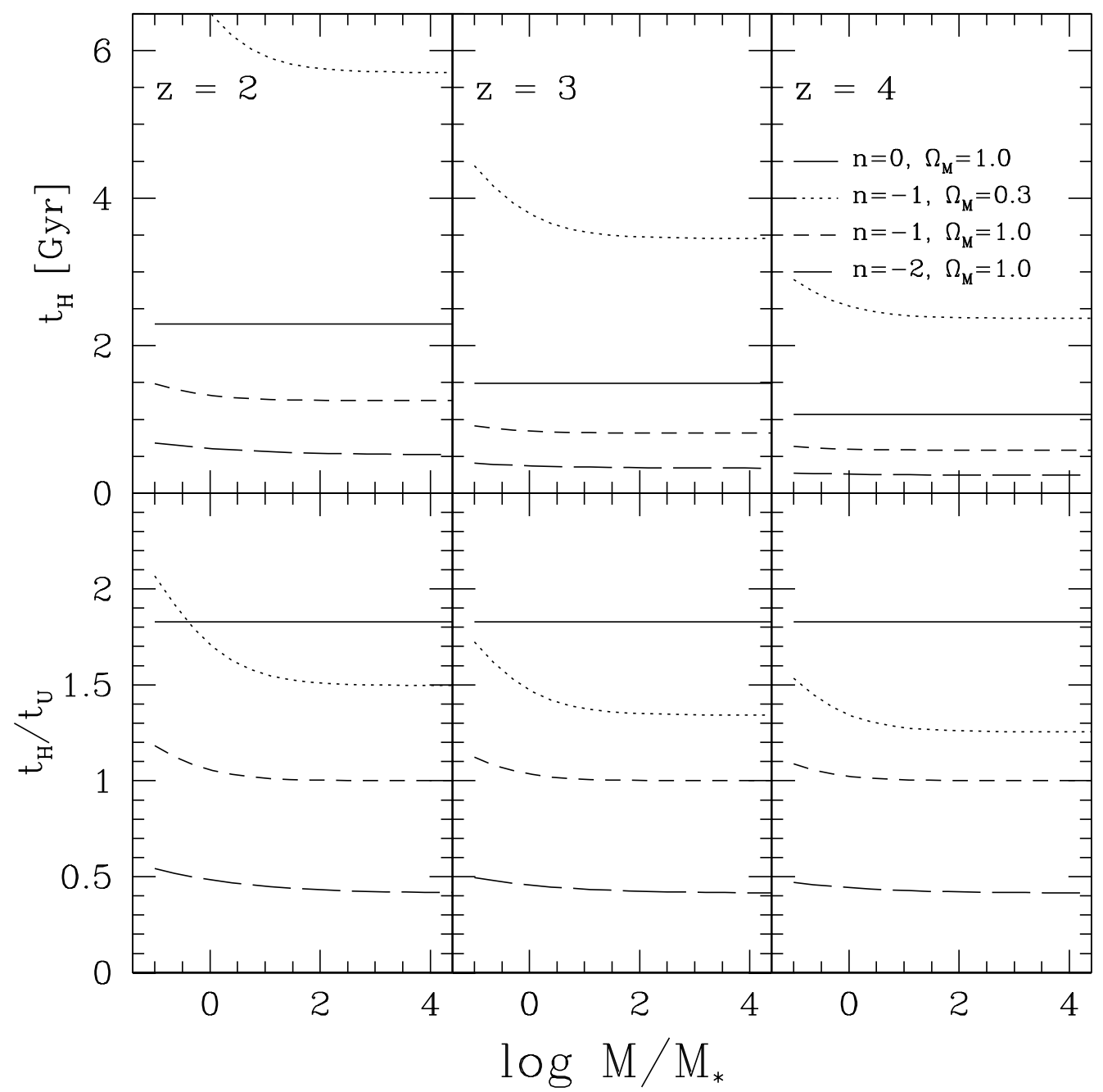

Fig. 1.-Halo lifetimes vs. $M / M_{*}$ for the power-law cosmologies listed in Table 1 . Top panels show the halo lifetimes in Gyr for each model at $z=2$, 3 , and 4. Bottom panels show the ratio of the halo lifetime to the age of the universe. This ratio is independent of redshift for the $\Omega_{M}=1$ models, but not for the $n=-1, \Omega_{M}=0.3$ model.

$\Omega_{M}=0.3, \Omega_{\Lambda}=0$. The cosmological parameters for all our models with power-law power spectra are summarized in Table 1.

The power-law scaling of the rms fluctuation amplitude, equation (15), allows the bias equation (12) to be written

$$
\begin{aligned}
b(M, z)=( & \left.+\frac{1}{\delta_{c, 0}}\left\{\left[\frac{M}{M_{*}(z)}\right]^{(3+n) / 3}-1\right\}\right) \\
& \times\left\{\frac{1}{2}\left[\frac{M}{M_{*}(z)}\right]^{-2(3+n) / 3}+1\right\}^{(0.06-0.02 n)} .
\end{aligned}
$$

Note that the second factor is very close to 1 for $M \geq M_{*}$. Figure 2 shows the bias and the corresponding numberweighted effective bias (eq. [13]) as a function of $M_{\min } / M_{*}$. For $M_{\min }>M_{*}$, the effective bias is only slightly larger than $b\left(M_{\min }\right)$, since the number density of halos declines rapidly with increasing $M$. As equation (18) shows, the bias depends more strongly on $M$ for larger $n$. However, the exponentially falling tail of the mass function at high $M / M_{*}$ is much steeper for higher $n$, as one can see from equation (16). As a result, the bias at fixed comoving number density is higher for smaller $n$ in the high $M / M_{*}$ regime (see Fig. 3 below).
Under the (good) approximation that the halo lifetime is given by the asymptotic formula (eq. [17]) in the mass range of interest, the halo lifetime can be moved outside the integral of equation (8) for the number density of active quasars. The implied quasar lifetime as a function of minimum halo mass is then

$$
t_{Q}\left(M_{\min }\right)=\frac{t_{H} \Phi(z)}{N\left(M>M_{\min }\right)},
$$

where $N\left(M>M_{\min }\right)$ is given by equation (16). For the $\Omega_{M}=0.3, n=-1$ model, we also use the asymptotic value of $t_{H}$, although this is no longer given by equation (17). We use a $P(k)$ normalization $\sigma_{8}=0.5$ for the three $\Omega_{M}=1$ models and $\sigma_{8}=1.0$ for the $\Omega_{M}=0.3$ model, in approximate agreement with the constraint on $\sigma_{8}$ and $\Omega_{M}$ implied by the observed mass function of rich galaxy clusters (White, Efstathiou, \& Frenk 1993; Eke, Cole, \& Frenk 1996).

Equation (19) implicitly determines $M_{\min } / M_{*}(z)$ given $t_{Q}$. The top panels of Figure 3 show $M_{\min } / M_{*}(z)$ as a function of $t_{Q} / t_{U}$ for $z=2,3$ and 4 and a constant comoving space density $\Phi(z)=10^{-6} h^{3} \mathrm{Mpc}^{-3}$. For the $\Omega_{M}=1$ cases, where $t_{Q} / t_{U}$ depends only on $n$ and $M_{\min } / M_{*}$, the redshift dependence of $M_{\min } / M_{*}$ arises solely from the presence of 
TABLE 1

Power-LaW Model Parameters

\begin{tabular}{|c|c|c|c|c|c|c|}
\hline $\begin{array}{c}n \\
(1)\end{array}$ & $\begin{array}{l}\sigma_{8} \\
(2)\end{array}$ & $\begin{array}{c}h \\
(3)\end{array}$ & $\begin{array}{r}\Omega_{M} \\
\text { (4) }\end{array}$ & $\begin{array}{c}M_{*}(z=2) \\
\quad(5)\end{array}$ & $\begin{array}{c}M_{*}(z=3) \\
(6)\end{array}$ & $\begin{array}{c}M_{*}(z=4) \\
(7)\end{array}$ \\
\hline $0 \ldots$ & 0.5 & 1.0 & 1.0 & $5.87 \times 10^{12}$ & $3.30 \times 10^{12}$ & $2.11 \times 10^{12}$ \\
\hline$-1 \ldots \ldots$ & 0.5 & 1.0 & 1.0 & $5.80 \times 10^{12}$ & $2.45 \times 10^{12}$ & $1.25 \times 10^{12}$ \\
\hline$-1 \ldots \ldots$ & 1.0 & 1.0 & 0.3 & $5.44 \times 10^{12}$ & $2.87 \times 10^{12}$ & $1.70 \times 10^{12}$ \\
\hline$-2 \ldots \ldots$ & 0.5 & 1.0 & 1.0 & $5.59 \times 10^{8}$ & $9.96 \times 10^{7}$ & $2.61 \times 10^{7}$ \\
\hline
\end{tabular}

NoTE.-Parameters of the four power-law cosmological models discussed in $\S 2.5$. Cols. (1)-(4) list the power spectrum index $n$, normalization $\sigma_{8}$, scaled Hubble constant $h$, and mass density parameters $\Omega_{M}$. Cols. (5)-(7) list the values of $M_{*}$ (eq.

[7]) at $z=2,3$, and 4, in units of $h^{-1} M_{\odot}$.

$M_{*}$ in the number density equation (16). As $M_{*}$ increases with decreasing redshift, the value of $x_{\min }=M_{\min } / M_{*}$ must decrease to keep $\Phi(z)$ constant. Smaller values of $n$ lead to higher values of $M_{\min } / M_{*}$ because of the gentler falloff of the mass function at large $M / M_{*}$. The $n=-2$ curves become flat at the largest values of $t_{Q} / t_{U}$ because $t_{Q}$ begins to exceed the halo lifetime $t_{H}$, implying that all halos above $M_{\min }$ are occupied by quasars. The difference between the open and $\Omega_{M}=1$ curves for $n=-1$ reflects mainly the larger values of $\sigma_{8}$ and $D(z)$ in the open model, which lead to a lower value of $\rho_{0} / M_{*}$ in the mass function (eq. [16]) and therefore require a lower value of $M_{\min } / M_{*}$ to compensate.

The middle panels of Figure 3 show the effective bias, $b_{\text {eff }}\left(M_{\text {min }}, z\right)$, for the power-law models. As already remarked, the bias at fixed space density and $t_{Q} / t_{U}$ is higher for redder power spectra (smaller $n$ ) because of the much higher values of $M_{\min } / M_{*}$, despite the partially counterbalancing effect of the stronger dependence of bias on mass at larger $n$. Physically, the higher bias for redder spectra reflects the greater influence of the large-scale environment on the amplitude of small-scale fluctuations. For a given

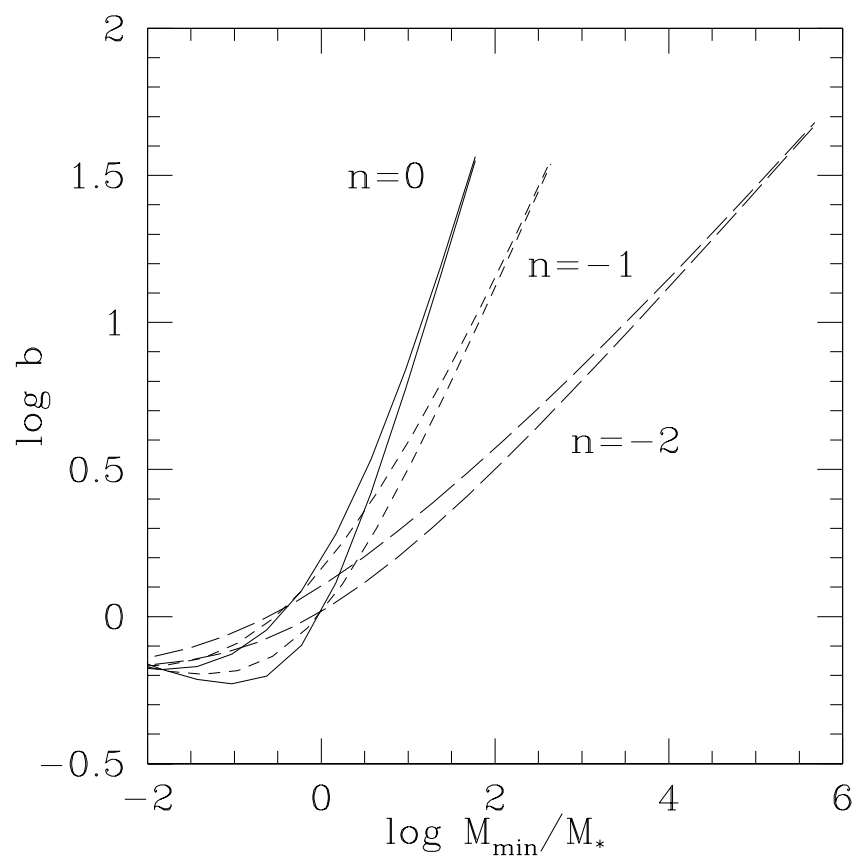

FIG. 2.-Bias vs. $M / M_{*}$ for the power-law models with $n=0$ (solid line), -1 (short-dashed line), and -2 (long-dashed line). Lower curves show $b\left(M_{\min }\right)$ computed from eq. (12), and upper curves show the numberweighted effective bias (eq. [13]). model, the bias increases with increasing redshift, reflecting the increase in $M_{\min } / M_{*}$; the change, however, is quite modest.

The rms number count fluctuation on comoving scale $r$ is

$$
\begin{aligned}
\sigma_{Q}(r, z) & =b_{\text {eff }}\left(M_{\min }, z\right) \sigma(r, z) \\
& =b_{\text {eff }}\left(M_{\min }, z\right) \sigma_{8} D(z)\left(\frac{r}{8 h^{-1} \mathrm{Mpc}}\right)^{-(3+n) / 2} .
\end{aligned}
$$

The quasar clustering length $r_{1}$ is the scale on which this rms fluctuation amplitude is unity,

$$
r_{1}=\left(8 h^{-1} \mathrm{Mpc}\right)\left[b_{\mathrm{eff}}\left(M_{\min }, z\right) \sigma_{8} D(z)\right]^{2 /(3+n)} .
$$

The bottom panels of Figure 3 present the main result of this section, the dependence of $r_{1}$ on quasar lifetime for our four power-law models at $z=2,3$, and 4 . As anticipated, the quasar clustering length shows a strong dependence on quasar lifetime. The relation between $r_{1}$ and $t_{Q}$ depends on the power spectrum index $n$, so the shape of the power spectrum must be known fairly well to determine $t_{Q}$ from measurements of $r_{1}$. The clustering at fixed $t_{Q} / t_{U}$ is substantially stronger in the open $n=-1$ model than in the $\Omega_{M}=$ 1 model, because the underlying mass distribution is more strongly clustered [larger $\sigma_{8}$ and $D(z)$ ].

For a specified value of $\Omega_{M}$, the cluster mass function imposes a reasonably tight constraint on the normalization, $\sigma_{8}$. It is nonetheless interesting to explore the sensitivity of the predicted quasar clustering to this normalization. More intuitive than the $\sigma_{8}$-dependence is the equivalent relation between the quasar clustering length and the corresponding clustering length of the underlying mass distribution at the same redshift,

$$
r_{1 m}=\left(8 h^{-1} \mathrm{Mpc}\right)\left[\sigma_{8} D(z)\right]^{2 /(3+n)} .
$$

Figure 4 plots this relation at $z=3$ for the four power-law cosmologies and $t_{Q}=t_{U}$ (top curve), $0.1 t_{U}, 0.01 t_{U}$, and $0.001 t_{U}$ (bottom curve), for values of $\sigma_{8}$ ranging from 0.2 to 2.0. The values of $r_{1 m}$ that correspond to the $\sigma_{8}$ values in Table 1 are marked with open circles. If the bias did not change with $r_{1 m}$, then the quasar clustering length $r_{1}$ would grow in proportion to $r_{1 m}$, and the curves in Figure 4 would parallel the diagonal of the box, which has a slope of 1.0. However, increasing $r_{1 m}$ increases $M_{*}$, and therefore requires a lower value of $M_{\min } / M_{*}$ to match the quasar space density. The correspondingly lower bias partially compensates for the larger $r_{1 m}$, making the curves in Figure 4 shallower than the box diagonal. For $n=0$ and large $t_{Q} / t_{U}$ (the highest solid curve), the minimum mass $M_{\min }$ lies far out on the tail of a steeply falling mass function. In this regime, a change in $M_{*}$ requires only a small change in 


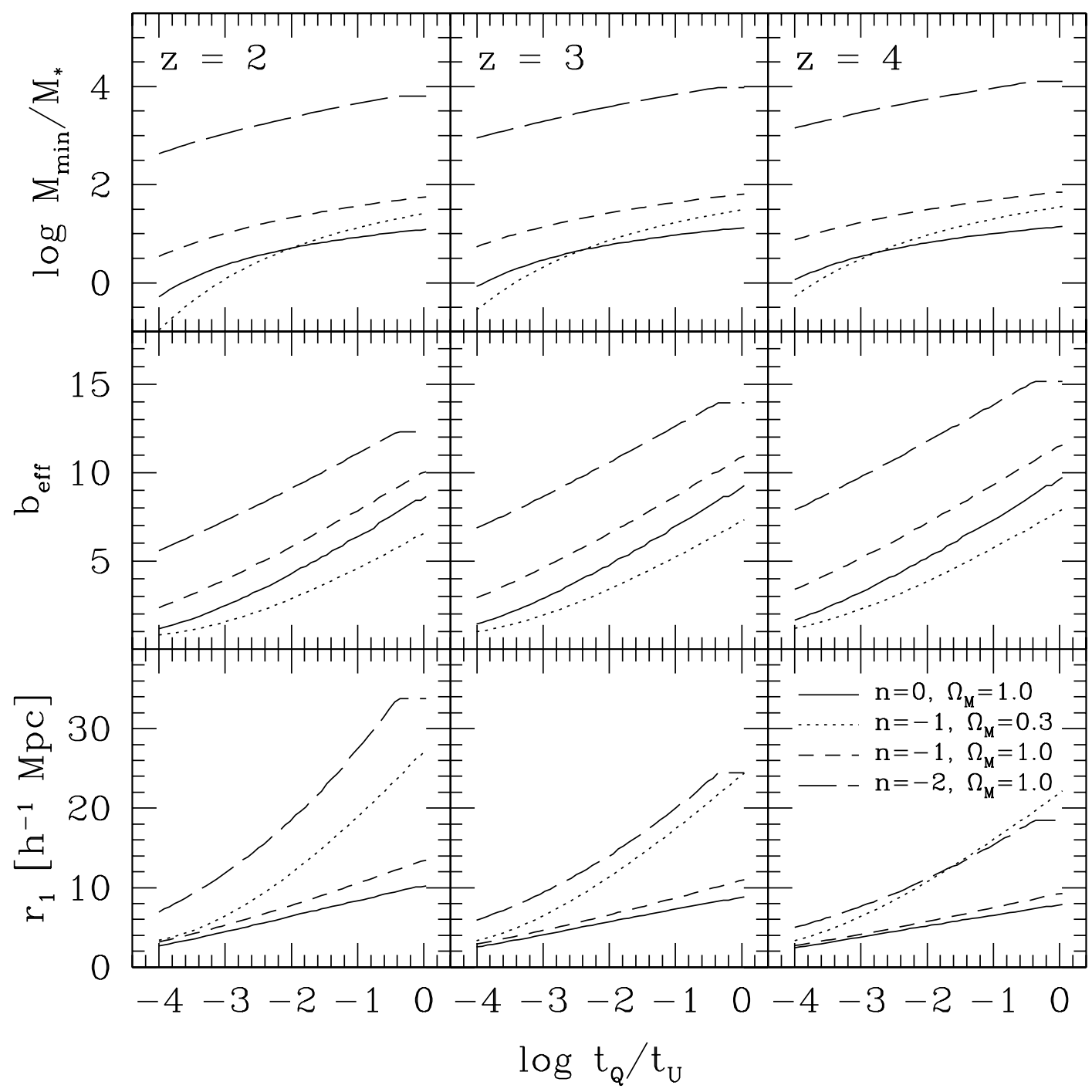

FIG. 3.- Halo mass, bias, and clustering length for the power-law models, as a function of quasar lifetime. Top panels show the minimum mass, $M_{\text {min }}$, required to obtain a space density of $\Phi(z)=10^{-6} h^{3} \mathrm{Mpc}^{-3}$ for a given value of $t_{Q} / t_{U}$ at $z=2,3$, and 4 . Middle panels show the corresponding effective bias, $b_{\text {eff }}$, for halos $M_{\min }$. Bottom panels show the clustering lengths, $r_{1}$, as a function of $t_{Q} / t_{U}$. The clustering length is the radius of a top-hat sphere in which rms number count fluctuations (in excess of Poisson) are unity. For a power-law correlation function $\xi(r)=\left(r / r_{0}\right)^{-1.8}, r_{1} \approx 1.4 r_{0}$.

$M_{\min } / M_{*}$ to compensate, so there is little change in $b_{\text {eff }}$ with $r_{1 m}$, and the curves approach the $r_{1} \propto r_{1 m}$ lines that would apply for constant bias. A similar argument explains the steepening of all curves toward low $r_{1 m}$, where the small values of $M_{*}$ put the value of $M_{\min }$ farther out on the tail of the mass function.

\section{RESULTS FOR COLD DARK MATTER COSMOLOGIES}

The results of $\S 2.5$ confirm our initial contention that quasar clustering can provide a good diagnostic of the typical quasar lifetime. However, they show that the predicted clustering length also depends on the shape of the mass power spectrum and on the value of $\Omega_{M}$, which influences the cluster normalization of $\sigma_{8}$ at $z=0$ and (together with $\Omega_{\Lambda}$ ) determines the growth factor $D(z)$. Accurate determination of $t_{Q}$ from measurements of quasar clustering therefore requires reasonably good knowledge of the underlying cosmology. Fortunately, many lines of evidence now point toward a flat, low-density model based on inflation and cold dark matter (see, e.g., the review by Bahcall et al. 1999). In particular, recent studies of the power spectrum of the Ly $\alpha$ forest imply that the matter power spectrum has the shape and amplitude predicted by $C O B E$ - and clusternormalized cold dark matter (CDM) models with $\Omega_{M} \sim 0.4$ at the redshifts and length scales relevant to the prediction of quasar clustering (Croft et al. 1999; Weinberg et al. 1999; McDonald et al. 2000; Phillips et al. 2000).

For the power spectrum of our CDM models, we adopt $P(k) \propto k^{n_{p}} T^{2}(k)$ with scale-invariant $\left(n_{p}=1\right)$ primeval inflationary fluctuations and the transfer function parameterization of Bardeen et al. (1986),

$$
\begin{aligned}
T(k)= & \frac{\ln (1+2.34 q)}{2.34 q}\left[1+3.89 q+(16.1 q)^{2}\right. \\
& \left.+(5.46 q)^{3}+(6.71 q)^{4}\right]^{-1 / 4} .
\end{aligned}
$$

Here $q=k / \Gamma$ and $\Gamma$, with units of $\left(h^{-1} \mathrm{Mpc}\right)^{-1}$, is the CDM shape parameter, given approximately by $\Gamma=$ $\Omega_{M} h \exp \left[-\Omega_{b}-(2 h)^{1 / 2} \Omega_{b} / \Omega_{M}\right]$ (Sugiyama 1995). We calculate $\sigma(M)$ and $(d \sigma / d M)$ by numerical integration of this power spectrum.

We consider five different CDM models with the parameters listed in Table 2. These models are chosen to illustrate a 


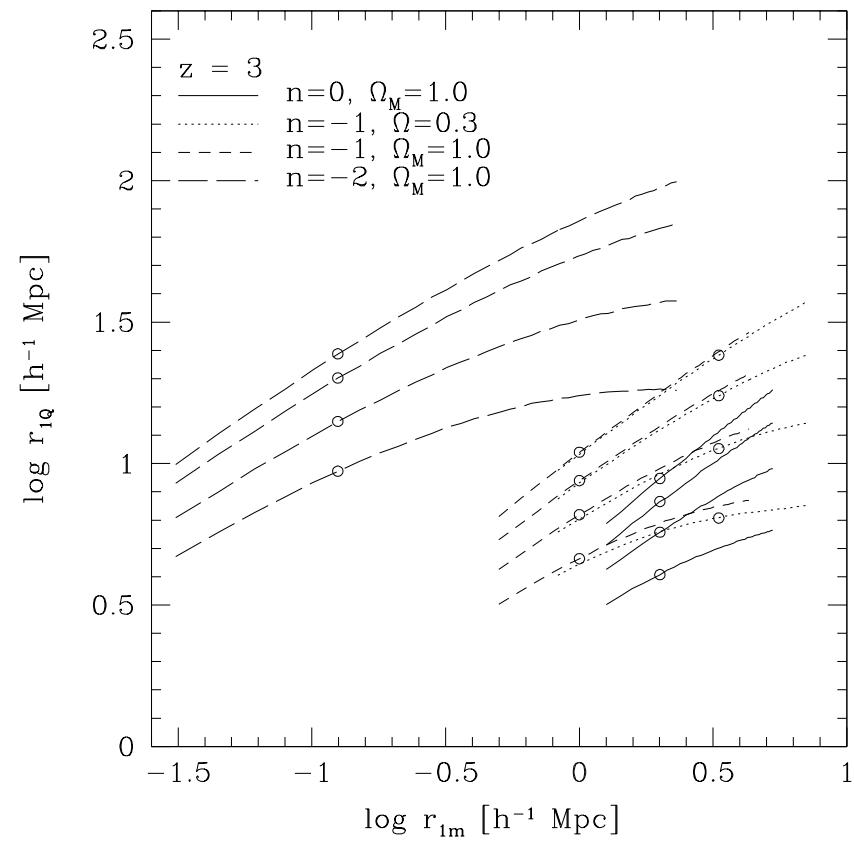

FIG. 4.-Dependence of the quasar clustering length, $r_{10}$, on the mass clustering length, $r_{1 m}$, for the four power-law models at $z=3$. In each case, the four lines show, from top to bottom, the lifetimes $t_{Q}=t_{U}, 0.1 t_{U}, 0.01 t_{U}$, and $0.001 t_{U}$. Results are computed for normalizations running from $\sigma_{8}=$ 0.2 to $\sigma_{8}=2.0$. Open circles show $r_{1 m}$ and $r_{1 Q}$ for our standard choices of $\sigma_{8}$, listed in Table 1 . If bias were independent of $r_{1 m}$, the lines would parallel the diagonal of the box, which has a slope of 1.0.

range of cosmological inputs and also to isolate the effects of different parameters on quasar clustering predictions. The $\tau \mathrm{CDM}, \mathrm{OCDM}$, and $\Lambda \mathrm{CDM}$ models have $\Gamma=0.2$, in approximate agreement with the shape parameter estimated from galaxy surveys (e.g., Baugh \& Efstathiou 1993; Peacock \& Dodds 1994), and they have $\sigma_{8}$ values consistent with the cluster mass function constraints of Eke et al. (1996). The $\tau$ CDM and $\Lambda$ CDM models are approximately $C O B E$-normalized. $C O B E$ normalization would imply a lower $\sigma_{8}$ for OCDM, but a slight increase in $n_{p}$ could raise $\sigma_{8}$ without having a large impact on the shape of $P(k)$ at the relevant scales. The OCDM and $\Lambda$ CDM models are consistent with the Ly $\alpha$ forest power spectrum measurements of Croft et al. (1999), but the $\tau$ CDM model is not. OCDM is inconsistent with the observed location of the first acoustic peak in the cosmic microwave background anisotropy spectrum (e.g., Miller et al. 1999; Melchiorri et al. 2000; Tegmark \& Zaldarriaga 2000), and of the three models, only $\Lambda \mathrm{CDM}$ is consistent with the Hubble diagram of Type Ia supernovae (Riess et al. 1998; Perlmutter et al. 1999).
We use the comparison between the $\tau \mathrm{CDM}$ and SCDM models, with $\Gamma=0.2$ and $\Gamma=0.5$, respectively, to illustrate the impact of the power-spectrum shape for fixed $\Omega_{M}$ and $\sigma_{8}$. The SCDM model is cluster-normalized, but its $\sigma_{8}=0.5$ is well below the value of $\sigma_{8} \approx 1.2$ implied by $C O B E$ for $n_{p}=1, \Gamma=0.5$ (e.g., Bunn \& White 1997). The OCDM and $\Lambda$ CDM models have the same $P(k)$ shape and the same $P(k)$ amplitude at $z=0$, but at high redshift the OCDM model has stronger fluctuations as a result of a larger $D(z)$. We therefore include the model $\Lambda \mathrm{CDM} 2$, which has $\sigma_{8}$ chosen to yield the same power spectrum amplitude as OCDM at $z=3$. Differences between OCDM and $\Lambda$ CDM2 isolate the impact of a cosmological constant for fixed high-redshift mass clustering.

Figure 5 shows $t_{H}$ in Gyr (top panels) and $t_{H} / t_{U}$ (bottom panels) as a function of $M / M_{*}$ for the CDM models at $z=2,3$, and 4 . In contrast to the power-law models shown in Figure 1, the ratio $t_{H} / t_{U}$ does not approach a constant value but instead increases at very large $M / M_{*}$. This increase can be understood with reference to the power-law case: the effective power-law index, $n_{\text {eff }}=3-6(d \ln \sigma /$ $d \ln M$ ), increases with increasing mass in a CDM spectrum, and larger values of $n_{\text {eff }}$ correspond to slower growth of mass scales (and larger $t_{H} / t_{U}$ ), as shown in Figure 1. The difference between the SCDM and $\tau$ CDM curves in Figure 5 reflects the higher values of $n_{\text {eff }}$ for the $\Gamma=0.5$ power spectrum. The differences between the various $\Gamma=0.2$ models largely reflect the differences in $M_{*}$, and hence the differences in $n_{\text {eff }}$ at fixed $M / M_{*}$, and they also reflect the differences in fluctuation growth rates.

Figure 6 plots the effective bias against $M_{\min } / M_{*}$ for the five CDM models at $z=3$. Figure 2 showed that the value of $b_{\text {eff }}$ at fixed $M_{\min } / M_{*}$ is higher for larger $n$. The lines in Figure 6 curve upward because $n_{\text {eff }}$ increases with mass scale, and to a good approximation the value of $b_{\text {eff }}$ in the CDM models equals the value of $b_{\text {eff }}$ at the same $M_{\min } / M_{*}$ in a power-law model of index $n_{\text {eff }}\left(M_{\text {min }}\right)$. The difference between SCDM and $\tau$ CDM in Figure 6 therefore reflects the higher $n_{\text {eff }}$ values in SCDM, and the differences among the other models reflect the different values of $M_{*}$, and hence the different values of $n_{\text {eff }}$ at fixed $M_{\min } / M_{*}$.

The top three panels of Figure 7 show the dependence of $M_{\min } / M_{*}$ on $t_{Q}$ at $z=2,3$, and 4 ; the values of $M_{*}$ are listed in Table 2 . The calculation of $M_{\min }$ via equation (8) incorporates both the dependence of halo lifetime on mass and the influence of $\Omega_{M}$ and $\Omega_{\Lambda}$ on the value of $\Phi(z)$ inferred from the quasar surface density (as discussed in the Appendix). The two $\Omega_{M}=1$ models have the lowest values of $M_{*}$ because of their lower $\sigma_{8}$ and $D(z)$, so they require the largest $M_{\min } / M_{*}$ to match the observed $\Phi(z)$. The value of

TABLE 2

CDM MODEL PARAMETERS

\begin{tabular}{ccccccccc}
\hline \hline $\begin{array}{c}\text { Model } \\
(1)\end{array}$ & $\begin{array}{c}\sigma_{8} \\
(2)\end{array}$ & $\begin{array}{c}h \\
(3)\end{array}$ & $\begin{array}{c}\Omega_{M} \\
(4)\end{array}$ & $\begin{array}{c}\Omega_{\Lambda} \\
(5)\end{array}$ & $\begin{array}{c}\Gamma \\
(6)\end{array}$ & $\begin{array}{c}M_{*}(z=2) \\
(7)\end{array}$ & $\begin{array}{c}M_{*}(z=3) \\
(8)\end{array}$ & $\begin{array}{c}M_{*}(z=4) \\
(9)\end{array}$ \\
\hline $\mathrm{SCDM} \ldots \ldots .$. & 0.5 & 0.5 & 1.0 & 0.0 & 0.5 & $3.58 \times 10^{9}$ & $2.26 \times 10^{8}$ & $1.86 \times 10^{7}$ \\
$\tau \mathrm{CDM} \ldots \ldots \ldots$ & 0.5 & 0.5 & 1.0 & 0.0 & 0.2 & $9.44 \times 10^{6}$ & $1.09 \times 10^{5}$ & $1.87 \times 10^{3}$ \\
$\mathrm{OCDM} \ldots \ldots$. & 0.9 & 0.65 & 0.3 & 0.0 & 0.2 & $1.50 \times 10^{11}$ & $2.70 \times 10^{10}$ & $5.60 \times 10^{9}$ \\
$\Lambda \mathrm{CDM} \ldots \ldots$. & 0.9 & 0.65 & 0.3 & 0.7 & 0.2 & $2.70 \times 10^{10}$ & $2.03 \times 10^{9}$ & $1.91 \times 10^{8}$ \\
$\Lambda \mathrm{CDM} 2 \ldots \ldots$ & 1.17 & 0.65 & 0.3 & 0.7 & 0.2 & $2.16 \times 10^{11}$ & $2.34 \times 10^{10}$ & $3.10 \times 10^{9}$ \\
\hline
\end{tabular}

NotE.-Parameters of the five CDM models discussed in $\S 3$. Col. (1) lists the model name, cols. (2)-(5) the power spectrum normalization and cosmological parameters, and col. (6) the power spectrum shape parameter (see eq. [23]). Cols. (7)-(9) list the values of $M_{*}$ (eq. [7]) at $z=2,3$, and 4, in units of $h^{-1} M_{\odot}$. 


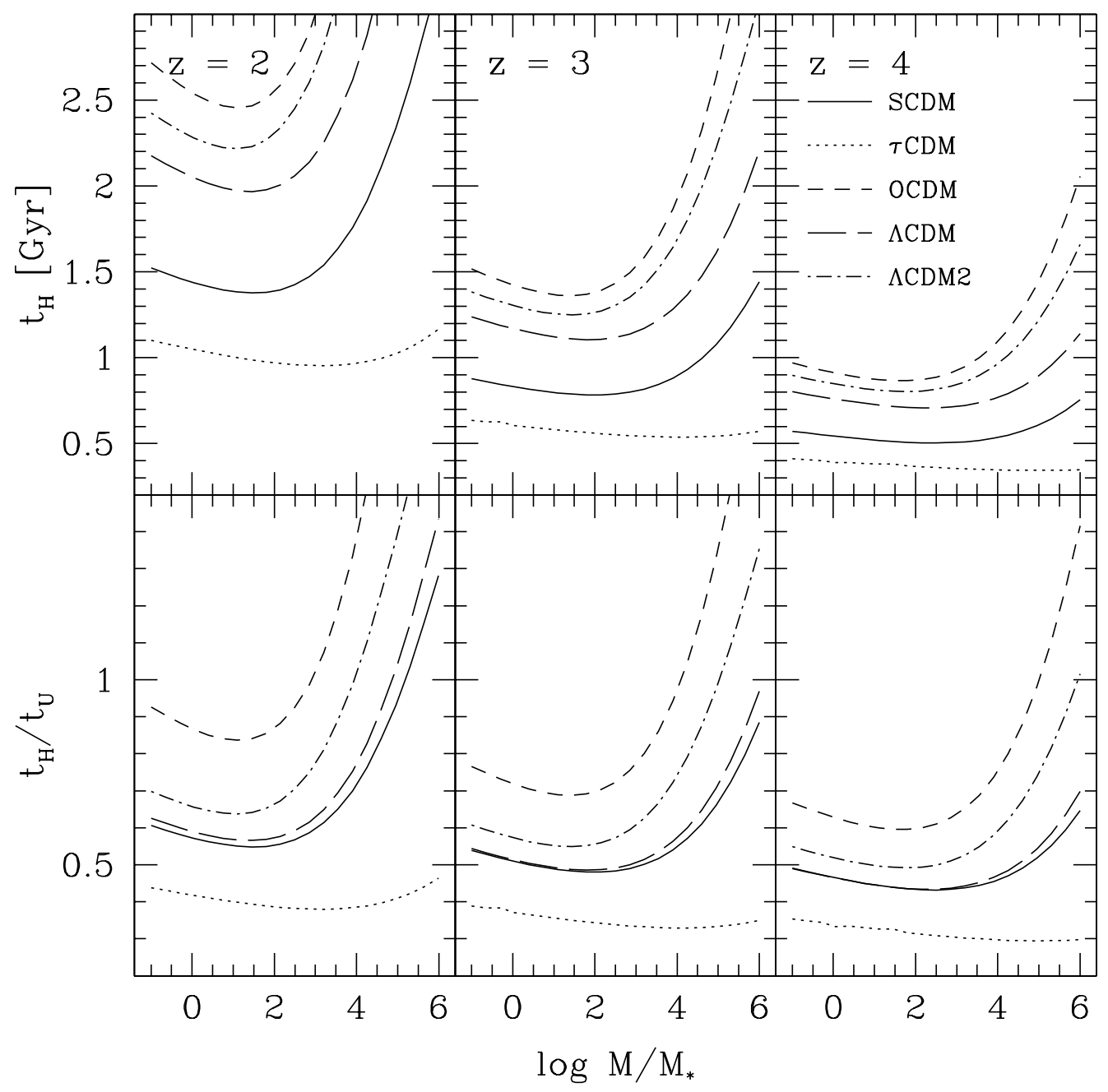

FIG. 5.-Halo lifetime as a function of $M / M_{*}$, as in Fig. 1, for the CDM models with parameters listed in Table 2

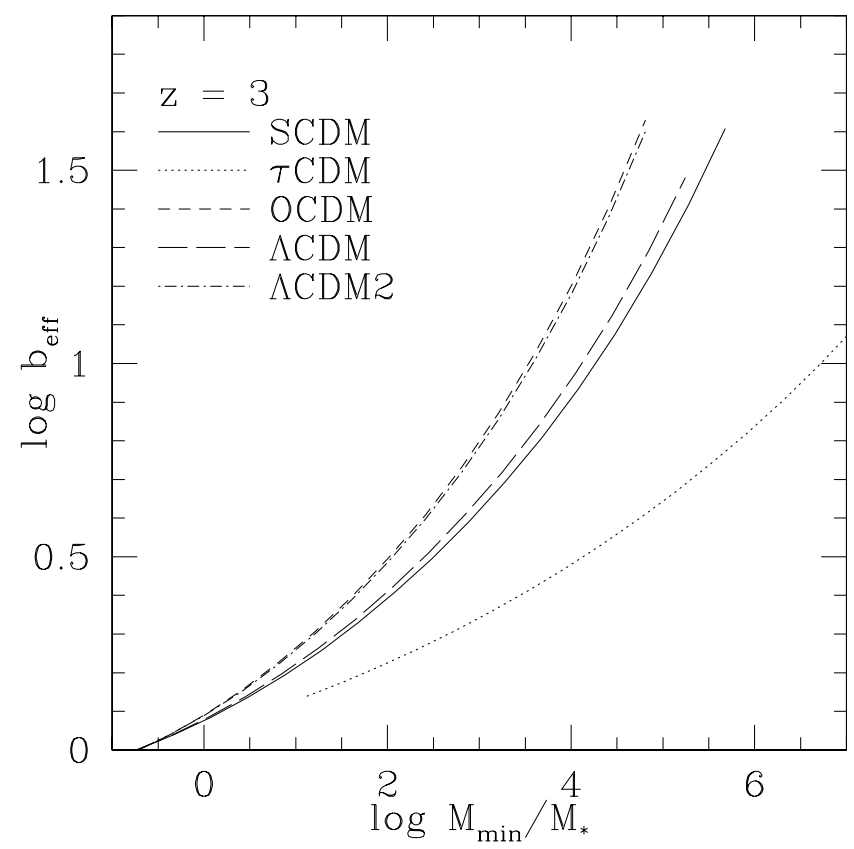

FIG. 6.-Effective bias as a function of minimum halo mass, as in Fig. 2, for the CDM models at $z=3$.
$M_{*}$ is smaller for $\Lambda$ CDM than for OCDM because $D(z)$ is smaller for the flat model, so $\Lambda \mathrm{CDM}$ requires larger $M_{\min } / M_{*}$. The higher normalization of the $\Lambda$ CDM2 model largely removes this difference, since $\sigma_{8} D(z=3)$ is matched to that of the OCDM model, but $\Lambda$ CDM 2 still has a slightly lower $M_{*}$ because of the influence of $\Omega_{\Lambda}$ on $\delta_{c}(z)$. As a result, the $M_{\min } / M_{*}$ curve for $\Lambda \mathrm{CDM} 2$ lies just above that of OCDM at $z=3$.

The middle panels of Figure 7 show the effective bias values, which display the same relative dependence on $t_{Q}$ and cosmology as the $M_{\min } / M_{*}$ values. Because $M_{\text {min }} / M_{*}>1$ in all the CDM models, even for $t_{Q}$ as low as $10^{5} \mathrm{yr}$, the MW bias formula (eq. [11]) yields nearly identical results to Jing's (1998) corrected formula (eq. [12]).

The bottom panels of Figure 7 present the main results of this paper: the relation between the clustering length $r_{1}$ and the quasar lifetime $t_{Q}$ for CDM models at $z=2,3$, and 4 . The clustering length is an increasing function of quasar lifetime for the reasons outlined in $\S 1$ and detailed in $\S 2$. A longer $t_{Q}$ implies that quasar host halos are rarer, more highly biased objects. The change in the $r_{1}$ versus $t_{Q}$ relation with redshift reflects the evolution of the quasar space density and of the underlying mass fluctuations. For a given model and $t_{Q}$, the predicted quasar clustering is weakest at 
No. 1, 2001

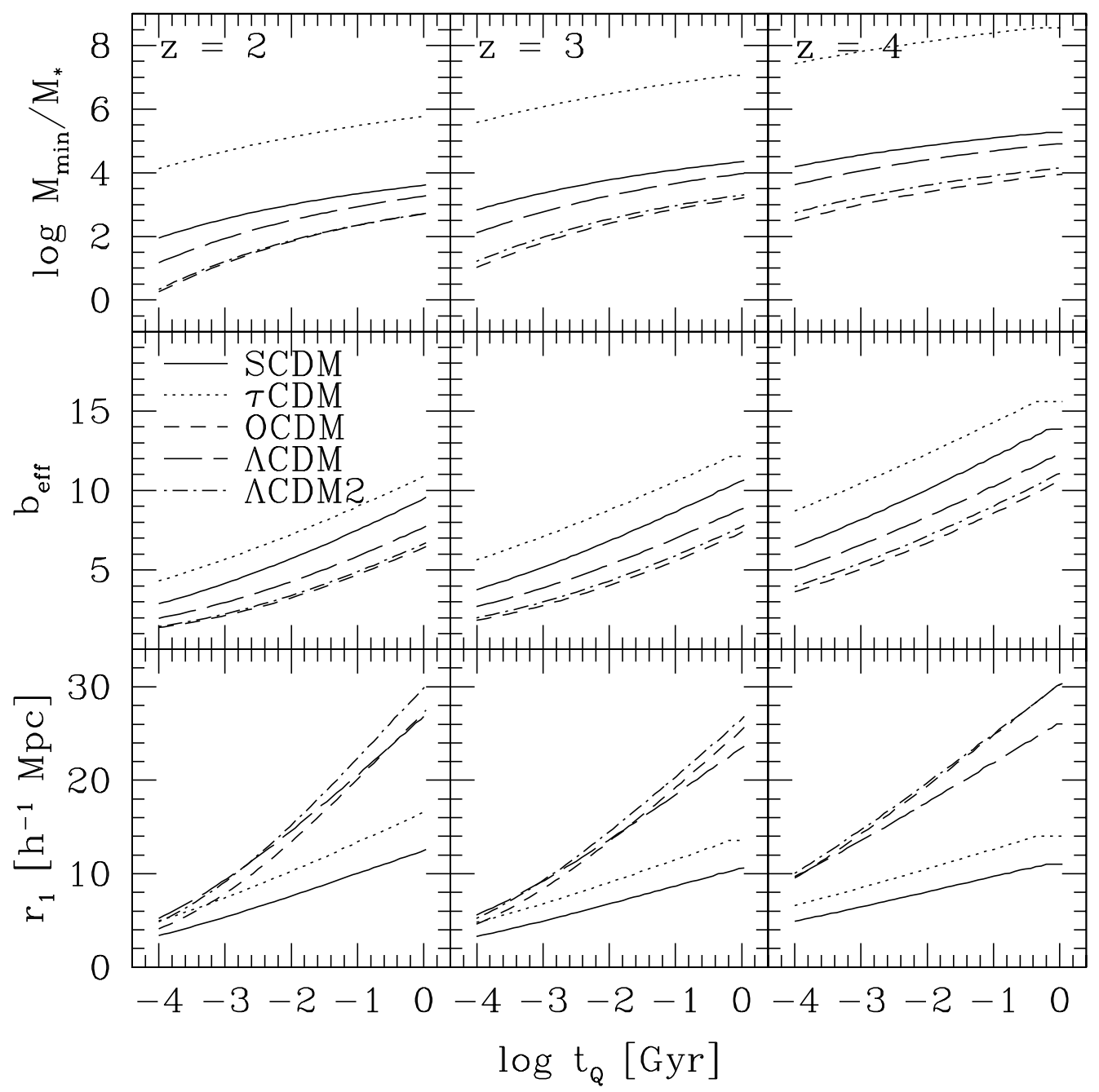

Fig. 7.- Minimum halo mass, effective bias, and clustering length as a function of $t_{Q}$, for the CDM models at $z=2,3$, and 4 . Format is the same as in Fig. 3.

$z=3$, the peak of the quasar space density. The smaller quasar abundance at $z=4$ implies a higher bias of the host halo population, which more than compensates for the slightly weaker mass clustering. The clustering length grows between $z=3$ and $z=2$ because of both the drop in quasar space density and the growth of mass clustering. The $r_{1}$ versus $t_{Q}$ relation becomes flat at the largest $t_{Q}$ for the SCDM model at $z=4$ and for the $\tau$ CDM model at $z=3$ and 4 , where $t_{Q}$ exceeds the halo lifetime $t_{H}\left(M_{\mathrm{min}}\right)$, and the value of $M_{\min }$ required to match $\Phi(z)$ therefore becomes independent of $t_{Q}$.

The differences between models reflect the differences in bias factors discussed above and the differences in the mass clustering. There are also differences in the values of $\Phi(z)$ inferred from the observed quasar surface density (see Appendix), but these have relatively little effect. The main separation in Figure 7 is between the low-density models and the $\Omega_{M}=1$ models, which have weaker mass clustering

TABLE 3

$r_{1}$ Vs. $t_{Q}$ FitTing CoEFFICIENTS

\begin{tabular}{|c|c|c|c|c|c|c|c|c|c|}
\hline \multirow[b]{2}{*}{$\begin{array}{l}\text { MoDEL } \\
\text { (1) }\end{array}$} & \multicolumn{3}{|c|}{$z=2$} & \multicolumn{3}{|c|}{$z=3$} & \multicolumn{3}{|c|}{$z=4$} \\
\hline & $\begin{array}{l}a_{0} \\
(2)\end{array}$ & $\begin{array}{l}a_{1} \\
(3)\end{array}$ & $\begin{array}{l}a_{2} \\
\text { (4) }\end{array}$ & $\begin{array}{l}a_{0} \\
(5)\end{array}$ & $\begin{array}{l}a_{1} \\
(6)\end{array}$ & $\begin{array}{l}a_{2} \\
(7)\end{array}$ & $\begin{array}{l}a_{0} \\
(8)\end{array}$ & $\begin{array}{l}a_{1} \\
(9)\end{array}$ & $\begin{array}{c}a_{2} \\
(10)\end{array}$ \\
\hline SCDM & 12.47 & 2.437 & 0.1001 & 10.62 & 1.932 & 0.0601 & 11.26 & 1.590 & -0.0042 \\
\hline$\tau \mathrm{CDM} \ldots \ldots \ldots$ & 16.54 & 3.163 & 0.1662 & 13.89 & 2.422 & 0.0882 & 14.48 & 1.964 & -0.0166 \\
\hline OCDM $\ldots . . .$. & 27.18 & 7.002 & 0.7798 & 25.32 & 6.029 & 0.5347 & 30.26 & 5.457 & 0.1640 \\
\hline$\Lambda \mathrm{CDM} \ldots \ldots$ & 26.79 & 6.173 & 0.4913 & 23.48 & 4.974 & 0.3122 & 26.12 & 4.248 & 0.0823 \\
\hline$\Lambda \mathrm{CDM} 2 \ldots \ldots$ & 29.84 & 7.441 & 0.7465 & 26.51 & 6.093 & 0.4840 & 30.23 & 5.274 & 0.1393 \\
\hline
\end{tabular}

NotE.-Coefficients for polynomial fits (eq. [24]) to the predicted relations between quasar lifetime and clustering length shown in Fig. 7. Col. (1) lists the model name; cols. (2)-(4) the coefficients for $z=2$; cols. (5)-(7) the coefficients for $z=3$; and cols. (8)-(10) the coefficients for $z=4$. 
because of their lower values of $\sigma_{8}$ and $D(z)$. The $\Omega_{M}=1$ models have larger bias factors, but these are not enough to compensate for the smaller mass fluctuations. The $r_{1}$ versus $t_{Q}$ relations are also shallower for the $\Omega_{M}=1$ models, because the values of $M_{\min }$ lie farther out on the steep, high-mass tail of the mass function, where a smaller change in $M_{\min }$ can make up for the same change in $t_{Q}$. The three low-density models yield very similar predictions.

To facilitate comparison of future observational results to our predictions, we have fitted polynomials of the form

$$
\begin{aligned}
& r_{1}= \\
& \begin{cases}a_{0}+a_{1} \log _{10} t_{Q} & \log _{10} t_{Q} \geq-1.5, \\
a_{0}+a_{1} \log _{10} t_{Q}+a_{2}\left(\log _{10} t_{Q}+1.5\right)^{2} & \log _{10} t_{Q}<-1.5,\end{cases}
\end{aligned}
$$

to each of the $r_{1}$ versus $t_{Q}$ curves shown in Figure 7. The values of the coefficients are given in Table 3 , and the coefficients $a_{0}$ and $a_{1}$ have the same value over the entire range in $t_{Q}$. These fits are accurate to better than $3 \%$ in $r_{1}$ for given $t_{Q}$, or to better than $10 \%$ in $t_{Q}$ given $r_{1}$, for all cases except the SCDM and $\tau \mathrm{CDM}$ models at $z=4$, where the maximum errors are $3 \%$ in $r_{1}$ given $t_{Q}$ and $20 \%$ in $t_{Q}$ for a given $r_{1}$.

\section{DISCUSSION}

\subsection{Sensitivity to Model Details}

As already mentioned in $\S 2.3$, the definition of a "halo lifetime" is somewhat ambiguous. We have so far adopted a definition of $t_{H}$ as the median time before a halo of mass $M$ is incorporated into a halo of mass $2 M$. If we increase this mass ratio from 2 to 5 (a rather extreme value), then the typical halo lifetimes in our CDM models increase by factors of 2-4. Since it is the ratio $t_{Q} / t_{H}$ that enters our determination of $M_{\min }$ (eq. [8]), and hence fixes the bias factor, this change in $t_{H}$ would require an equal increase in $t_{Q}$ to maintain the same clustering length $r_{1}$. We conclude that the ambiguity in halo lifetime definition introduces a factor of $\sim 2$ uncertainty in the determination of $t_{Q}$ from clustering measurements, in the context of our model.

We have also assumed that quasar luminosity is perfectly correlated with host halo mass, so that matching the space density of an absolute-magnitude-limited sample imposes a sharp cutoff in the host mass distribution at $M=M_{\min }$. If there is some scatter in the luminosity-host mass relation, then some halos with $M<M_{\min }$ will host a quasar above the absolute-magnitude limit and some halos with $M>$ $M_{\min }$ will not. We can model such an effect by introducing a soft cutoff into equation (8):

$$
\Phi(z)=\int_{0}^{\infty} d M g(M) \frac{t_{Q}}{t_{H}(M, z)} n(M, z)
$$

where

$$
\begin{aligned}
& g(M)= \\
& \begin{cases}0 & \text { for } M<\frac{M_{\min }}{\alpha} \\
\left(\frac{\alpha}{M_{\min }\left(\alpha^{2}-1\right)}\right) M-\frac{1}{\alpha^{2}-1} & \text { for } \frac{M_{\min }}{\alpha}<M<\alpha M_{\min } \\
1 & \text { for } M>\alpha M_{\min }\end{cases}
\end{aligned}
$$

and $\alpha>1$. Adopting a soft cutoff slightly decreases $M_{\text {min }}$ and, more significantly, reduces the value of $b_{\text {eff }}$ by allowing some quasars to reside in lower mass halos, which are less strongly biased. Quantitatively, we find that setting $\alpha=2$, which corresponds to including halos down to $M=M_{\min } / 2$, decreases the clustering length by $\lesssim 6 \%$ for the shortest quasar lifetimes and $\lesssim 10 \%$ for the longest quasar lifetimes. Matching a fixed $r_{1}$ with an $\alpha=2$ cutoff requires lifetimes that are longer by a factor of $\sim 1-1.5$ at short $t_{Q}$ and $\sim 2-2.5$ at long $t_{Q}$. Longer lifetimes are more sensitive to scatter in the luminosity-host mass relation because $b_{\text {eff }}$ depends more strongly on $M_{\min } / M_{*}$ for these rarer objects. The assumption of a perfectly monotonic relation between quasar luminosity and host mass leads to the smallest $t_{Q}$ for a given $r_{1}$. Thus, if any scatter does exist in this relation, our model predictions for $t_{Q}$ effectively become lower limits to the quasar lifetime.

Another simplification of our model is the assumption that a quasar is either "on" or "off"; each quasar shines at luminosity $L$ for time $t_{Q}$, perhaps divided among several episodes of activity, and the rest of the time it is too faint to appear in a luminous quasar sample. More realistically, variations in the accretion rate and radiative efficiency will cause the quasar luminosity to vary, especially if the black hole mass itself grows significantly during the luminous phase. Nonetheless, the maximum luminosity will still depend on the maximum black hole mass. At a given time, the luminous quasar population will include black holes shining at close to their maximum luminosity and "faded" black holes of higher mass. Because the host halos lie on the steeply falling tail of the mass function, the first component of the population always dominates over the second, and we therefore expect our clustering method to yield the time $t_{Q}$ for which a quasar shines within a factor $\sim 2$ of its peak luminosity. More strongly faded quasars are too rare to make much difference to the space density or effective bias.

To illustrate this point, we consider the model of Haehnelt et al. (1998) in which a quasar hosted by a halo of mass $M$ has a luminosity history $L(t)=L_{0}(M) \exp \left(-t / t_{Q}\right)$, with a maximum luminosity $L_{0}(M)=\alpha M$ proportional to the halo mass. In this model, the time that a quasar shines above the luminosity threshold, $L_{\min }=L_{0}\left(M_{\min }\right)$, of a survey is the visibility time, $t_{Q}^{\prime}=t_{Q} \ln \left(M / M_{\min }\right)$. We can calculate $M_{\min }$ for a given space density by substituting $t_{Q}^{\prime}$ for $t_{Q}$ in equation (8), then calculate $b_{\text {eff }}\left(M_{\min }\right)$ by multiplying the integrands in the numerator and denominator of equation (13) by the visibility weighting factor $\ln \left(M / M_{\min }\right)$. The middle curves in Figure 8 compare $r_{1}\left(t_{Q}\right)$ for the on-off (solid line) and exponential decay (dotted line) models, in the case of $\Lambda \mathrm{CDM}$ at $z=3$ with our standard $\Phi(z)$. The curves are remarkably similar, showing that the lifetime inferred from clustering assuming an on-off model would be close to the $e$-folding timescale in an exponential decay model. The curves for the exponential decay model are slightly shallower because at low $M_{\min }\left(\right.$ low $\left.t_{Q}\right)$ the mass function is not as steep, allowing faded quasars in more massive halos to make a larger contribution to $b_{\text {eff }}$ and thereby raise $r_{1}$. Although results for a different functional form of $L(M, t)$ would differ in detail, we would expect the lifetime inferred from clustering to be close to the "half-maximum" width of the typical luminosity history, for the general reasons discussed above.

As mentioned in $\S 2.1$, we assume that quasars radiate isotropically. If they radiate instead with an average 


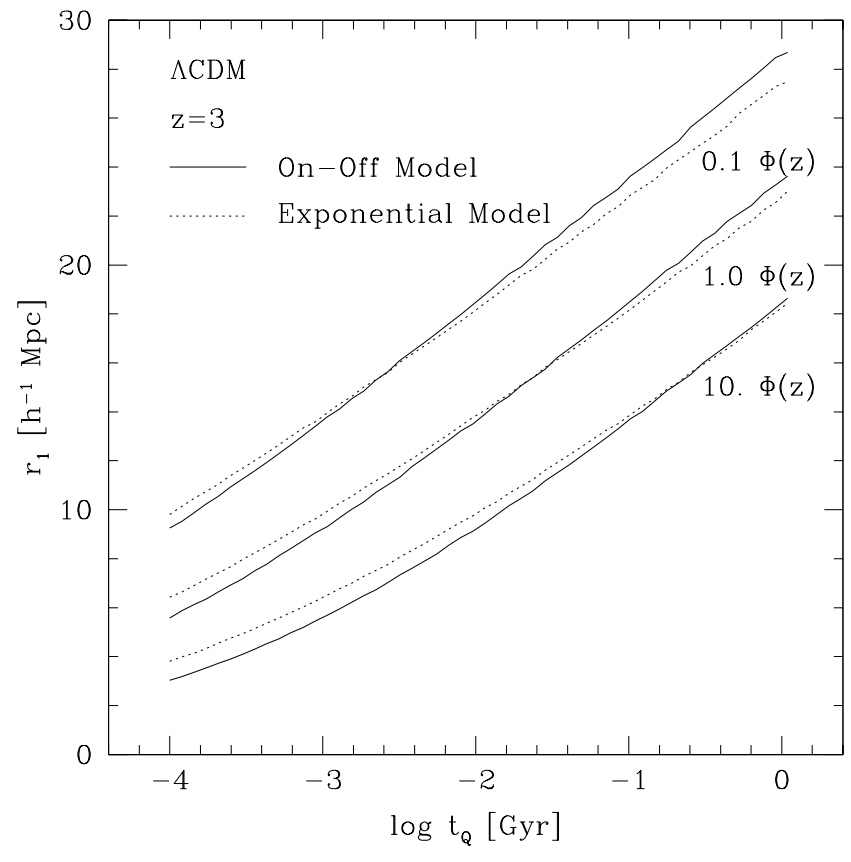

FIG. 8. - Clustering length vs. $t_{Q}$ for the $\Lambda$ CDM model at $z=3$ for two different models of quasar luminosity evolution at three different space densities $\Phi(z)$. The "on-off" model (solid lines) assumes that the quasar luminosity is constant throughout its lifetime $t_{Q}$ and is the standard model we discuss in this paper. The central line shows results for the $\Lambda \mathrm{CDM}$ model at $z=3$ with our standard $\Phi(z)$. The other solid curves, related to the first by simple horizontal shifts, show results for space densities different by factors of 10 and $1 / 10$ (bottom and top, respectively). In the exponential model (dotted lines), the quasar luminosity starts at some maximum luminosity proportional to the halo mass and decays with an $e$-folding timescale $t_{Q}$. The middle line again corresponds to our standard $\Phi(z)$, and the other two dotted curves show results for space densities different by factors of 10 and 1/10 (bottom and top, respectively).

beaming factor $f_{B}<1$, then the true value of $\Phi(z)$ is larger than the observed value by a factor $f_{B}^{-1}$. The implied lifetime for a given $r_{1}$ would therefore be larger by a factor $f_{B}^{-1}$ as well.

\subsection{Interpretation of Existing Data}

After several attempts (Osmer 1981; Webster 1982), quasar clustering was first detected by Shaver (1984), and later by Shanks et al. (1987) and Iovino \& Shaver (1988). However, measurements of quasar clustering are still hampered by small, sparse samples, and even the best studies to date yield detections with only several $\sigma$ significance. Given the limitations of current data, it is not surprising that different authors reach different conclusions about the strength of clustering and its evolution. Analyzing a combined sample of quasars with $0.3<z<2.2$ from the Durham/AAT UVX Survey, the Canada-France-Hawaii Telescope survey, and the Large Bright Quasar Sample, Shanks \& Boyle (1994) and Croom \& Shanks (1996) find a reasonable fit to the data with an $\Omega_{M}=1$ model that has $\xi(r)=\left(r / r_{0}\right)^{-\gamma}, \gamma \approx 1.8$, and a constant comoving correlation length $r_{0}=6 h^{-1} \mathrm{Mpc}$. La Franca, Andreani, \& Cristiani (1998) report a higher correlation length, $r_{0}=9.1$ $\pm 2.0 h^{-1} \mathrm{Mpc}$, for a $\gamma=1.8$ power law, in their $1.4<z<2.2$ sample.

If we adopt $r_{0} \approx 8 h^{-1} \mathrm{Mpc}$ at $z=2$ and a corresponding $r_{1} \approx 11 h^{-1} \mathrm{Mpc}$, then the implied quasar lifetime is $\sim 10^{7.5}$ $\mathrm{yr}$ for the $\tau \mathrm{CDM}$ model and $\sim 10^{8} \mathrm{yr}$ for SCDM. The $r_{0}$ values quoted above are for $\Omega_{M}=1$, and because quasar pair separations are measured in angle and redshift, they should be increased by a factor of $\sim 1.5$ in an $\Omega_{M}=0.3$, $\Omega_{\Lambda}=0.7$ universe and a factor of $\sim 1.3$ in an $\Omega_{M}=0.3$, $\Omega_{\Lambda}=0$ universe (roughly the inverse cube roots of the volume ratios listed in Table A1). Adopting $r_{1} \approx 16 h^{-1}$ Mpc implies a lifetime of $t_{Q} \sim 10^{7}-10^{7.5} \mathrm{yr}$ in our lowdensity models. However, these numbers must be considered highly uncertain because of the limitations of current data and because the space densities of the various observational samples do not necessarily match those assumed in our model predictions.

All these measurements are based mainly on quasars with $z<2$. At higher redshift, Kundić (1997) and Stephens et al. (1997) have investigated clustering in the Palomar Transit Grism Survey (PTGS; Schneider, Schmidt, \& Gunn 1994). Fitting a $\gamma=1.8$ power law, Stephens et al. (1997) find $r_{0}=$ $17.5 \pm 7.5 h^{-1} \mathrm{Mpc}$ for $z>2.7$. This high correlation length (inferred from the presence of three close pairs in a sample of 90 quasars) could be a statistical fluke, but in the context of our model it is tempting to see it as a consequence of the high luminosity threshold of the PTGS survey, which might lead it to pick out the most strongly clustered members of the quasar population.

\subsection{Prospects}

The 2dF (Boyle et al. 2000; Shanks et al. 2000) and Sloan (York et al. 2000) quasar surveys will transform the study of quasar clustering over the next several years, yielding highprecision measurements for a wide range of redshifts. These measurements will allow good determination of the typical quasar lifetime, $t_{Q}$, in the context of the model presented here. They will also test the key assumption of this model, the monotonic relation between quasar luminosity and host halo mass, by characterizing the clustering as a function of redshift and, especially, as a function of quasar absolute magnitude.

Figure 8 illustrates this test for the $\Lambda \mathrm{CDM}$ model at $z=3$. Brighter quasars have a lower space density $\Phi(z)$, so they should have a higher minimum host halo mass $M_{\min }$, and, because of the higher bias of more massive halos, they should exhibit stronger clustering. Fainter, more numerous quasars should exhibit weaker clustering. Figure 8 shows the predicted $r_{1}$ versus $t_{Q}$ relation for samples with $1 / 10$ and 10 times the space density of our standard case $(3.42$ quasars per $\mathrm{deg}^{2}$ per unit redshift; see Table A1). In our standard on-off model (solid lines), a change in $\Phi(z)$ in equation (8) can be exactly compensated for by changing $t_{Q}$ by the same factor, so the solid curves in Figure 8 are simply shifted horizontally relative to each other. Our predictions in Figure 7 (see eq. [24]) can therefore be transformed to any quasar space density by changing $t_{Q}$ in proportion to $\Phi(z)$. In the exponential decay model (dotted lines), the scaling of $t_{Q}$ with $\Phi(z)$ is no longer exact, although it is still a good approximation.

If there is a large dispersion in the relation between quasar luminosity and host halo mass, then the dependence of clustering strength on quasar space density will be much weaker than Figure 8 predicts. Detection of the predicted trend between luminosity and clustering, or a definitive demonstration of its absence, would itself provide an important insight into the nature of quasar host halos. More generally, the parameters of a model that incorporates 
scatter (such as the $\alpha$ prescription of eq. [26]) could be determined by matching the observed relation between $r_{1}$ and $\Phi(z)$.

If the observations do support a tight correlation between luminosity and host halo mass, then the first property of quasars to emerge from the $2 \mathrm{dF}$ and Sloan clustering studies will be the typical lifetime $t_{Q}$. For the low-density models in Figure 7, the slope of the correlation between $r_{1}$ and $\log _{10} t_{Q}$ is $\sim 10$, so a determination of $r_{1}$ with a precision of $2 h^{-1} \mathrm{Mpc}$ would constrain $t_{Q}$ to a factor of $10^{0.2} \approx 1.6$, for a specified cosmology. By the time these quasar surveys are complete, a variety of observations may have constrained cosmological parameters to the point that they contribute negligible uncertainty to this constraint. Instead, the uncertainty in $t_{Q}$ will probably be dominated by the limitations of the quasar population model, e.g., the approximate nature of the assumptions that the quasar luminosity tracks the halo mass, that there is only one quasar per halo, and that the average lifetime $t_{Q}$ is independent of quasar luminosity. These assumptions can be tested empirically to some degree, but not perfectly. Despite these limitations, it seems realistic to hope that $t_{Q}$ can be constrained to a factor of 3 or better by highprecision clustering measurements, a vast improvement over the current situation. It is worth reiterating that our assumption of a perfectly monotonic relation between luminosity and halo mass leads to the smallest $t_{Q}$ for an observed $r_{1}$, since with a shorter lifetime there are simply not enough massive, highly biased halos to host the quasar population.

A determination of $t_{Q}$ to a factor of 3 will be sufficient to address fundamental issues regarding the physics of quasars and galactic nuclei. Comparison of $t_{Q}$ to the Salpeter timescale will answer one of the most basic questions about supermassive black holes: do they shine as they grow? If $t_{Q} \gtrsim 4 \times 10^{7} \mathrm{yr}$, the $e$-folding timescale for $L \sim L_{\mathrm{E}}, \epsilon \sim 0.1$, then quasar black holes increase their mass by a substantial factor during their optically bright phase. If $t_{Q}$ is much shorter than this, then the black holes must accrete most of their mass at low efficiency, or while shining at $L \ll L_{\mathrm{E}}$. A short lifetime could indicate an important role for advection-dominated accretion (Narayan, Mahadevan, \& Quatert 1998 and references therein), or it could indicate that black holes acquire much of their mass through mergers with other black holes, emitting binding energy in the form of gravitational waves rather than electromagnetic waves. A determination of $t_{Q}$ would also resolve the question of whether the black holes in the nuclei of local galaxies are the remnants of dead quasars. For example, Richstone et al. (1998) infer a lifetime of $t_{Q} \sim 10^{6} \mathrm{yr}$ by matching the space density of local spheroids that host black holes of mass $M \gtrsim 4 \times 10^{8} M_{\odot}$ to the space density of high-redshift quasars of luminosity $L_{\mathrm{E}}(M) \gtrsim 6 \times 10^{46} \mathrm{ergs} \mathrm{s}^{-1}$. If clustering implies a much longer lifetime, then these numerous local black holes may once have powered active nuclei, but they were not the engines of the luminous, rare quasars.

We have assumed in our model that quasar activity is a random event in the life of the parent halo. Quasar activity might instead be triggered by a major merger, by a weaker "fly-by" interaction, or by the first burst of star formation in the host galaxy. Regardless of the trigger mechanism, the lifetime will be the dominant factor in determining the strength of high-redshift quasar clustering, if our assumed link between luminosity and halo mass holds. However, different triggering mechanisms might be diagnosed by more subtle clustering properties, such as features in the correlation function at small separations, or higher order correlations. At low redshift, where the evolution of the quasar population is driven by fueling rather than by black hole growth, the nature of the triggering mechanism might play a major role in determining quasars' clustering properties. The calculations presented here illustrate the promise of quasar clustering as a tool for testing ideas about quasar physics, a promise that should be fulfilled by the large quasar surveys now under way.

We thank James Bullock, Alberto Conti, Jordi MiraldaEscudé, Patrick Osmer, and Simon White for helpful discussions. We also thank the referee for constructive suggestions, which led to our consideration of the exponential decay model in $\S 4$. As we were nearing completion of this work, we learned of a similar, independent study by Z. Haiman \& L. Hui (Haiman \& Hui 2000); our general conclusions are consistent with theirs, although the approaches are quite different in detail, precluding a precise comparison of results. This work was supported in part by NSF grant AST 96-16822 and NASA grant NAG5-3525.

\section{APPENDIX A}

\section{CONVERTING FROM OBSERVED QUASAR NUMBERS TO $\Phi(z)$}

The observed quantity that is measured in studies of quasar clustering and the quasar space density is the number of sources brighter than a given apparent magnitude $m$ per unit redshift per unit solid angle on the sky. This surface density per unit redshift can be converted into a comoving space density of objects brighter than a given absolute magnitude $M$,

$$
\Phi(z,<M)=\frac{d N(<m)}{d \Omega d z} \frac{d \Omega d z}{d V_{c}(z)},
$$

where $d V_{c}(z)$ is the differential comoving volume element corresponding to $d \Omega d z$. Following the notation in Hogg (1999), this volume element is

$$
d V_{c}(z)=D_{H} \frac{D_{M}^{2}}{E(z)} d \Omega d z
$$


TABLE A1

Quasar Space and Surface Density

\begin{tabular}{ccccc}
\hline \hline$z$ & $\Phi(z)$ & $\frac{d N}{d z d \Omega}$ & $\frac{f(1.0,0.0)}{f(0.3,0.0)}$ & $\frac{f(1.0,0.0)}{f(0.3,0.7)}$ \\
$(1)$ & $(2)$ & $(3)$ & $(4)$ & $(5)$ \\
\hline $2 \ldots \ldots \ldots \ldots \ldots$ & $1.889 \times 10^{-6}$ & 2.132 & 0.425 & 0.279 \\
$3 \ldots \ldots \ldots \ldots \ldots$ & $3.331 \times 10^{-6}$ & 3.417 & 0.339 & 0.253 \\
$4 \ldots \ldots \ldots \ldots \ldots$ & $3.200 \times 10^{-7}$ & 0.287 & 0.287 & 0.241 \\
\hline
\end{tabular}

NotE.-Adopted values of the space density of quasars at $z=2,3$, and 4, and cosmological conversion factors. Values of $\Phi(z)$ in col. (2) are from Warren et al. 1994 (assuming $\Omega_{M}=1$ ) for quasars with $M_{c}<-24.5$ (absolute continuum flux at $1216 \AA$ ), converted from their adopted $h=0.75$ to $h^{3} \mathrm{Mpc}^{-3}$. Col. (3) lists the abundance of quasars in number per $\operatorname{deg}^{2}$ per unit redshift to which the space density in col. (2) corresponds. Cols. (4) and (5) contain the ratios of the factors $f\left(\Omega_{M}, \Omega_{\Lambda}\right)$ defined in eq. (A6) needed to convert the space density in col. (2), which is valid for $\Omega_{M}=1$, to space densities for the OCDM and $\Lambda$ CDM models, respectively.

where $D_{H}=c / H_{0}$ is the Hubble distance, $D_{M}$ is the transverse comoving distance,

$$
D_{M}= \begin{cases}D_{H} \frac{1}{\sqrt{\Omega_{k}} \sinh \left(\sqrt{\Omega_{k}} \frac{D_{c}}{D_{H}}\right)} & \text { for } \Omega_{k}>0 \\ D_{H} \int_{0}^{z} \frac{d z^{\prime}}{E\left(z^{\prime}\right)} & \text { for } \Omega_{k}=0 \\ D_{H} \frac{1}{\sqrt{\left|\Omega_{k}\right|}} \sin \left(\sqrt{\left|\Omega_{k}\right|} \frac{D_{c}}{D_{H}}\right) & \text { for } \Omega_{k}<0\end{cases}
$$

and $E(z)=\left[\Omega_{M}(1+z)^{3}+\Omega_{k}(1+z)^{2}+\Omega_{\Lambda}\right]^{1 / 2}$, where $\Omega_{k}=1-\Omega_{M}-\Omega_{\Lambda}$. For $\Omega_{M}=1$ and $\Omega_{k}=0$, the differential comoving volume element is

$$
d V_{C}(z)=4\left(\frac{c}{H_{0}}\right)^{3}(1+z)^{-3 / 2}\left(1-\frac{1}{\sqrt{1+z}}\right)^{2} d \Omega d z
$$

per steradian per unit redshift.

The fact that $d V_{c}(z)$ depends on the cosmological parameters means that a given measured surface density of sources corresponds to a different comoving space density for different cosmological model parameters. The space density of quasars is commonly quoted for an $\Omega_{M}=1$ universe. To convert this space density (in units of $h^{3} \mathrm{Mpc}^{-3}$ ) into the space density for a model with different values of $\Omega_{M}$ and $\Omega_{\Lambda}$ requires a correction of the form

$$
\Phi\left(z, \Omega_{M}, \Omega_{\Lambda}\right)=\Phi\left(z, \Omega_{M}^{\prime}, \Omega_{\Lambda}^{\prime}\right) \frac{f\left(z, \Omega_{M}^{\prime}, \Omega_{\Lambda}^{\prime}\right)}{f\left(z, \Omega_{M}, \Omega_{\Lambda}\right)}
$$

where

$$
f\left(z, \Omega_{M}, \Omega_{\Lambda}\right)=\frac{D_{H} D_{M}^{2}}{E(z)} .
$$

This procedure converts the reported space density under one set of cosmological parameters back into the observed surface density and then converts the surface density into the space density for the new set of cosmological parameters. In the notation of Popowski et al. (1998), $f\left(z, \Omega_{M}, \Omega_{\Lambda}\right)=g f^{2}$, where $f$ and $g$ are given by their equations (5) and (6), respectively.

In Table A1 we list the factors to convert the space density in column (2), which is listed for $\Omega_{M}=1, \Omega_{\Lambda}=0$, to the corresponding space densities for $\Omega_{M}=0.3, \Omega_{\Lambda}=0.0$ and $\Omega_{M}=0.3, \Omega_{\Lambda}=0.7$. The factors in Table A1 are all less than unity because the comoving volume element is smallest in an $\Omega_{M}=1$ universe.

\section{REFERENCES}

Adelberger, K. L., Steidel, C. C., Giavalisco, M., Dickinson, M., Pettini, M., \& Kellogg, M. 1998, ApJ, 505, 18

Bahcall, N. A., Ostriker, J. P., Perlmutter, S., \& Steinhardt, P. J. 1999, Science, 284, 1481

Bajtlik, S., Duncan, R. C., \& Ostriker, J. P. 1988, ApJ, 327, 570

Bardeen, J., Bond, J. R., Kaiser, N., \& Szalay, A. S. 1986, ApJ, 304, 15

Baugh, C. M., \& Efstathiou, G. 1993, MNRAS, 265, 145

Bechtold, J. 1994, ApJS, 91, 1
Bond, J. R., Cole, S., Efstathiou, G., \& Kaiser, N. 1991, ApJ, 379, 440

Boyle, B. J., Fong, R., Shanks, T., \& Peterson, B. A. 1990, MNRAS, 243, 1

Boyle, B. J., Shanks, T., Croom, S. M., Smith, R. J., Miller, L., Loaring, N., \& Heymans, C. 2000, MNRAS, 317, 1014

Boyle, B. J., Smith, R. J., Shanks, T., Croom, S. M., \& Miller, L. 1999, in IAU Symp. 183, Cosmological Parameters and the Evolution of the Universe, ed. K. Sato (Dordrecht: Kluwer), 178

Bunn, E. F., \& White, M. 1997, ApJ, 480, 6 
Carroll, S. M., Press, W. H., \& Turner, E. L. 1992, ARA\&A, 30, 499

Ciotti, L., \& Ostriker, J. P. 1999, ApJ, submitted (preprint astro-ph/ 9912064)

Croft, R. A. C., Weinberg, D. H., Pettini, M., Katz, N., \& Hernquist, L. 1999, ApJ, 520, 1

Croom, S. M., \& Shanks, T. 1996, MNRAS, 281, 893

Efstathiou, G., \& Rees, M. J. 1988, MNRAS, 230, 5P

Eke, V. R., Cole, S., \& Frenk, C. S. 1996, MNRAS, 282, 263

Faber, S. M., \& Jackson, R. E. 1976, ApJ, 204, 668

Fan, X., et al. 1999, AJ, 118, 1

Haehnelt, M. G., Natarajan, P., \& Rees, M. J. 1998, MNRAS, 300, 817

Haehnelt, M. G., \& Rees, M. J. 1993, MNRAS, 263, 168

Haiman, Z., \& Hui, L. 2000, ApJ, in press (preprint astro-ph/0002190)

Haiman, Z., \& Loeb, A. 1998, ApJ, 503, 505

Heath, D. J. 1977, MNRAS, 179, 351

Hewett, P. C., Foltz, C. B., \& Chaffee, F. C. 1993, ApJ, 406, L43

Hogg, D. W. 1999, preprint (astro-ph/9905116)

Iovino, A., \& Shaver, P. A. 1988, ApJ, 330, L13

Jing, Y. P. 1998, ApJ, 503, L9

Kaiser, N. 1984, ApJ, 284, L9

Katz, N., Hernquist, L., \& Weinberg, D. H. 1999, ApJ, 523, 463

Katz, N., Quinn, T., Bertschinger, E., \& Gelb, J. M. 1994, MNRAS, 270, L71

Kauffmann, G., \& Haehnelt, M. 2000, MNRAS, 311, 576

Kolatt, T. S., et al. 1999, ApJ, 523, L109

Kundić, T. 1997, ApJ, 482, 631

Lacey, C., \& Cole, S. 1993, MNRAS, 262, 627

La Franca, F., Andreani, P., \& Cristiani, S. 1998, ApJ, 497, 529

Lynden-Bell, D. 1969, Nature, 223, 690

Magorrian, J., et al. 1998, AJ, 115, 2285

McDonald, P., Miralda-Escudé, J., Rauch, M., Sargent, W. L. W., Barlow, T. A., Cen, R., \& Ostriker, J. P. 2000, ApJ, 543, 1

Melchiorri, A., et al. 2000, ApJ, 536, L63

Miller, A. D., et al. 1999, ApJ, 524, L1

Mo, H. J., Mao, S., \& White, S. D. M. 1999, MNRAS, 304, 175

Mo, H. J., \& White, S. D. M. 1996, MNRAS, 282, 347 (M-W)

Monaco, P., Salucci, P., \& Danese, L. 2000, MNRAS, 311, 279

Narayan, R., Mahadevan, R., \& Quatert, E. 1998, in The Theory of Black Hole Accretion Discs, ed. M. A. Abramowicz, G. Bjornsson, \& J. E. Pringle (Cambridge: Cambridge Univ. Press), 148
Navarro, J. F., Frenk, C. S., \& White, S. D. M. 1997, ApJ, 490, 493

Osmer, P. S. 1981, ApJ, 247, 762

1998, in ASP Conf. Ser. 146, The Young Universe, ed.

S. D'odorico, A. Fontana, \& E. Giallongo (San Francisco: ASP), 1

Peacock, J. A., \& Dodds, S. J. 1994, MNRAS, 267, 1020

Peebles, P. J. E. 1980, The Large Scale Structure of the Universe (Princeton: Princeton Univ. Press)

Perlmutter, S., et al. 1999, ApJ, 517, 565

Phillips, J., Croft, R. A. C., Weinberg, D. H., Hernquist, L., Katz, N., \& Pettini, M. 2000, ApJ, submitted (preprint astro-ph/0001089)

Press, W. H., \& Schechter, P. 1974, ApJ, 187, 425 (P-S)

Popowski, P. A., Weinberg, D. H., Ryden, B. S., \& Osmer, P. S. 1998, ApJ, 498,11

Richstone, D., et al. 1998, Nature, 395, A14

Riess, A. G., et al. 1998, AJ, 116, 1009

Salpeter, E. E. 1964, ApJ, 140, 796

Salucci, P., Szuszkiewicz, E., Monaco, P., \& Danese, L. 1999, MNRAS, 307,637

Schneider, D. P., Schmidt, M., \& Gunn, J. E. 1994, AJ, 107, 1245

Shanks, T., \& Boyle, B. J. 1994, MNRAS, 271, 753

Shanks, T., Boyle, B. J., Croom, S. M., Loaring, N., Miller, L., \& Smith, R. J. 2000, in ASP Conf. Ser. 200, Clustering at High Redshift, ed. A. Mazure, O. LeFevre, \& V. Lebrun (San Francisco: ASP), in press

Shanks, T., Fong, R., Boyle, B. J., \& Peterson, B. A. 1987, MNRAS, 227, 739

Shaver, P. A. 1984, A\&A, 136, L9

Stephens, A. W., Schneider, D. P., Schmidt, M., Gunn, J. E., \& Weinberg, D. H. 1997, AJ, 114, 41

Sugiyama, N. 1995, ApJS, 100, 281

Tegmark, M., \& Zaldarriaga, M. 2000, ApJ, 544, 30

Turner, E. L. 1991, AJ, 101, 5

van der Marel, R. P. 1999, AJ, 117, 744

Warren, S. J., Hewett, P. C., \& Osmer, P. S. 1994, ApJ, 421, 412

Webster, A. 1982, MNRAS, 199, 683

Weinberg, D. H., Croft, R. A. C., Hernquist, L., Katz, N., \& Pettini, M. 1999, ApJ, 522, 563

White, S. D. M., Efstathiou, G. P., \& Frenk, C. S. 1993, MNRAS, 262, 1023

York, D., et al. 2000, AJ, in press

Zeldovich, Ya. B., \& Novikov, I. D. 1964, Soviet Phys. Dokl., 158, 811 